\title{
๖The Role of the Divergent Circulation for Large-Scale Eddy Momentum Transport in the Tropics. Part I: Observations
}

\author{
PABLO ZURITA-GOTOR \\ Universidad Complutense de Madrid, and Instituto de Geociencia UCM-CSIC, Madrid, Spain
}

(Manuscript received 2 October 2018, in final form 10 January 2019)

\begin{abstract}
This work investigates the role played by the divergent circulation for meridional eddy momentum transport in the tropical atmosphere. It is shown that the eddy momentum flux in the deep tropics arises primarily from correlations between the divergent eddy meridional velocity and the rotational eddy zonal velocity. Consistent with previous studies, this transport is dominated by the stationary wave component, associated with correlations between the zonal structure of the Hadley cell (zonal anomalies in the meridional overturning) and the climatological-mean Rossby gyres. This eddy momentum flux decomposition implies a different mechanism of eddy momentum convergence from the extratropics, associated with upper-level mass convergence (divergence) over sectors with anomalous westerlies (easterlies). By itself, this meridional transport would only increase (decrease) isentropic thickness over regions with anomalous westerly (easterly) zonal flow. The actual momentum mixing is due to vertical (cross isentropic) advection, pointing to the key role of diabatic processes for eddy-mean flow interaction in the tropics.
\end{abstract}

\section{Introduction}

This paper is concerned with the dynamics of meridional eddy momentum transport in the tropical troposphere. While early paradigms of tropical momentum transport envisioned a nearly inviscid Hadley cell decelerated by Rossby waves of extratropical origin, Lee (1999) showed that in fact, the westerly eddy forcing compensates the deceleration by the off-equatorial Hadley cell in the deep tropics. As this eddy forcing plays a key role in theories of equatorial superrotation (Hide 1969), the maintenance of the tropical momentum budget has received much attention in idealized studies of planetary atmospheres (Showman and Polvani 2011; Pinto and Mitchell 2014; Laraia and Schneider 2015). It has also been suggested that the terrestrial atmosphere might transition to a superrotating state in a much warmer climate (Caballero and Huber 2010).

Dima et al. (2005) performed a detailed analysis of the tropical momentum budget in the terrestrial atmosphere

¿ Denotes content that is immediately available upon publication as open access.

\footnotetext{
Corresponding author: Pablo Zurita-Gotor, pzurita@ alum.mit.edu
}

and found the eddy forcing to be dominated by the stationary meridional momentum convergence. They proposed that the observed momentum transport could be associated with the atmospheric response to asymmetric tropical heating as in the classical Gill (1980) problem, though they did not study the dynamics of this response in detail. There is also modeling evidence from idealized studies (Suarez and Duffy 1992; Saravanan 1993; Kraucunas and Hartmann 2005) that asymmetric tropical heating produces a westerly acceleration in the deep tropics and can lead to superrotation when the Hadley cell upwelling is not too far off the equator (Kraucunas and Hartmann 2005). In all these studies, the posited mechanism for the tropical acceleration is the meridional propagation of Rossby waves forced by the heating, similar to the dynamics of the extratropical eddy-driven jet. An alternative perspective was provided by Showman and Polvani (2011), who studied the dynamics of equatorial acceleration in Gill-like settings motivated by the superrotation of tidally locked planets. These authors noted some problems with the classical paradigm of jet driving by meridionally propagating Rossby waves in the tropics, and proposed an alternative mechanism based on the interaction between the Kelvin and Rossby components of the Matsuno-Gill response. Zurita-Gotor and Held (2018) have recently argued that 
the same mechanism could drive the superrotation of nonconvecting atmospheres at large thermal Rossby number, except that the Kelvin and Rossby waves would be forced by an ageostrophic instability in this case (Iga and Matsuda 2005; Wang and Mitchell 2014).

A fundamental concept in our understanding of wave-mean flow interaction is the link between eddy dissipation and mean-flow acceleration (Andrews and McIntyre 1976). Zurita-Gotor and Held (2018) identify two different routes to eddy dissipation in planetary atmospheres that can lead to the spinup of eddy-driven jets. The first mechanism involves dissipation by breaking vorticity waves (i.e., vorticity mixing along isentropic surfaces). This produces an easterly acceleration over the breaking region (if the vorticity gradient is positive), which is compensated by westerly acceleration over the source region when these two regions are distinct. This mechanism is thought to be responsible for the spinup of the extratropical jet (Vallis 2006) and may also give rise to tropical eddy-driven jets in the presence of a robust tropical vorticity source (Suhas et al. 2017). Alternatively, the dissipation driving the mean flow change could be diabatic. The latter appears to be the underlying mechanism for superrotation in the simulations of Showman and Polvani (2011) and Zurita-Gotor and Held (2018), in which the tropical vorticity fluxes are weak and/or negative, and the westerly acceleration is imparted by vertical (cross isentropic) momentum advection instead.

The weakness of the Rossby wave source in the deep tropics (Sardeshmukh and Hoskins 1988) and the diabatic character of the tropical circulation suggest that the second mechanism might also be more relevant for eddy momentum forcing in the terrestrial atmosphere. Our study aims to test this conjecture and to investigate its implications for eddy momentum transport in the tropical troposphere more broadly. We will show that since the divergent flow is an important component of tropical eddy momentum transport, the mechanism of meridional momentum transport is different from that in the extratropics. We will also investigate the role of the different components of the climatological-mean tropical circulation in the momentum transport and characterize the momentum transport by the MaddenJulian oscillation (MJO). We present a comprehensive description of the observed tropical momentum flux in this paper while a companion study (Zurita-Gotor 2019, manuscript submitted to J. Atmos. Sci., hereafter Part II) will investigate the dynamical determinants of this momentum flux.

This paper is organized as follows. Section 2 introduces the data and methodology used. Section 3 provides some context by reviewing the seasonally varying tropical momentum balance and the impact of eddy forcing for angular momentum conservation and superrotation. Section 4 performs a meridional-vertical decomposition of the eddy momentum forcing using the advective and flux forms. It is shown that while meridional propagation dominates, the momentum mixing occurs primarily in the vertical near the equator. Section 5 discusses two different mechanisms that can produce meridional eddy momentum convergence, which are relevant for tropical and extratropical eddy-driven jets, respectively. Section 6 analyzes the dominant contributions to the meridional eddy momentum flux, and section 7 focuses on the MJO. We close with a brief summary and some discussion in section 8 .

\section{Data and methods}

We use in this study four-times daily gridded wind data $(u, v, \omega)$ at $2.5^{\circ}$ horizontal resolution from ERAInterim (Dee et al. 2011) for the 37-yr period spanning from 1979 to 2016. For some of our analyses, the horizontal wind field $(u, v)$ is decomposed into its nondivergent (rotational) and irrotational (divergent) components, which will be denoted using $r$ and $d$ subscripts in the following. The rotational wind is defined in terms of the streamfunction $\psi$, obtained by inversion of the vorticity field $\xi$, and the divergent wind is defined in terms of the velocity potential $\chi$, obtained by inversion of the divergence field $D$.

Eddy components, denoted with primes, are defined as deviations from zonal averages (indicated by overbars), and eddy fluxes are computed by zonally averaging the products of the daily mean eddy anomalies. Seasonal cycles of individual variables and eddy fluxes are defined in terms of their daily climatologies over the 37-yr record. For display purposes, this daily climatology is smoothed using a 20-day running mean.

For each calendar month, we define the stationary eddy component of a variable as the 37-yr monthly mean eddy climatology for that variable. Seasonal cycles of stationary eddy fluxes are then computed by zonally averaging the products of these stationary eddy components for each month. We also define seasonal cycles in the transient eddy fluxes as the differences between the seasonal cycles in the full eddy fluxes (averaged for each calendar month) and in the stationary eddy fluxes. These transient eddy fluxes include variability at both submonthly and interannual time scales, but some of the slow intraseasonal variability may be contaminated into the seasonal variability due to the reduced record. This could affect in particular the MJO variability, with periods on the order of a month. 

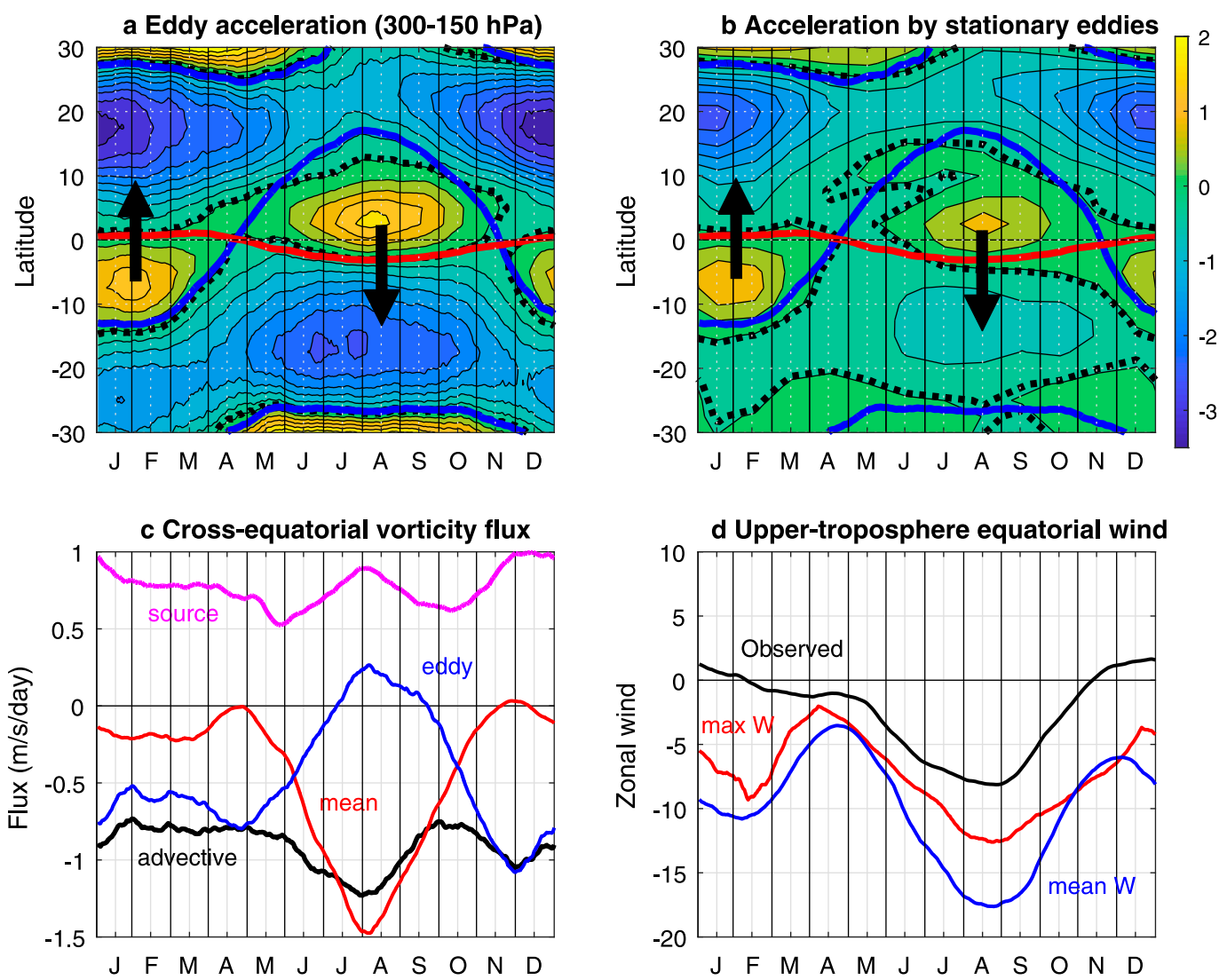

FIG. 1. (a) Seasonal cycle of net eddy acceleration $\left(\mathrm{m} \mathrm{s}^{-1}\right.$ day $\left.{ }^{-1}\right)$, with the zero contour shown with a thick black dotted line. The red line shows the vorticity equator (latitude with $f+\bar{\xi}=0$ ) and the blue line the latitude of zero meridional flow. The arrows indicate the directions of the seasonal Hadley cells. (b) As in (a), but for the acceleration by the stationary eddies. (c) Cross-equatorial vorticity flux (in acceleration units: $\mathrm{m} \mathrm{s}^{-1}$ day $^{-1}$ ) by the Hadley cell (red), by the eddies (blue), and net advective vorticity flux (black). Also shown with magenta line is the NH vorticity generation by vertical advection and tilting. (d) Observed equatorial zonal wind (black) and equatorial wind predicted by angular momentum conservation based on the planetary momentum at the latitude of maximum zonal-mean upwelling (red) or the weighted average of planetary momentum over the regions of mean tropical upwelling (blue). All variables are integrated over the 300-150-hPa layer and daily climatologies have been smoothed using a 20-day running mean.

To investigate the impact of the MJO in more detail, we use regression analysis based on the MJO indices of Adames and Wallace (2014). These authors define the MJO cycle in terms of the two leading global EOFs of the velocity potential difference between 150 and $850 \mathrm{hPa}$, computed after removing the seasonal cycle and lowfrequency $(T>120$ days) variability from the data. The two leading EOFs (with similar explained variances) represent structures in near quadrature, and the corresponding principal components are very highly correlated at lags of about a week, as expected for a propagating mode of variability. Regressions for the different MJO phases are computed as linear combinations of the regressions on the two standardized principal components characterizing the in-quadrature MJO phases. We perform independent analyses for the extended winter [November-March (NDJFM)] and summer [June-September (JJAS)] seasons following Adames et al. (2016), and assess statistical significance using a conservative decorrelation time scale of 10 days to estimate the number of degrees of freedom (Adames and Wallace 2014).

\section{The seasonally varying tropical momentum balance}

Figure 1a shows the seasonal cycle of the net [meridional plus vertical; see Eq. (3) in the next section] uppertroposphere zonal-mean eddy acceleration, vertically averaged between 300 and $150 \mathrm{hPa}$. As noted by Dima et al. (2005), tropical eddies transport momentum from the winter to the summer hemisphere against the Hadley 
cell meridional flow (illustrated with arrows). The eddy forcing in Fig. 1a includes contributions by both transient and stationary eddies. The stationary component dominates the full eddy forcing during DJF but the transient eddy forcing is also important during JJA (Fig. 1b).

Throughout the seasonal cycle, the transition between westerly and easterly eddy acceleration is well captured by the thick blue and red lines, which mark the latitudes where the meridional flow $\bar{v}$ and the absolute vorticity $f+\bar{\xi}$ (both integrated over the $150-300$-hPa layer) vanish. This is consistent with the approximate momentum balance:

$$
-(f+\bar{\xi}) \bar{v} \approx S,
$$

where $S$ is the eddy acceleration. We neglected in this balance vertical momentum advection by the Hadley cell, which can be locally important over the regions of zonal-mean tropical upwelling. This term is important at the equator during both equinox seasons and also explains the differences between the blue and zero lines during boreal summer.

The above balance requires that the meridional momentum flux is maximized near the "vorticity equator," which we define as the latitude where $f+\bar{\xi}=0$ (red line). This latitude is associated with the maximum zonal-mean angular momentum. Figure 1a shows that the vorticity equator shifts into the winter hemisphere during the seasonal cycle, leaving the equator over a region of westerly eddy acceleration. The shift is significantly stronger during JJA than during DJF. The seasonal migration of the vorticity equator is consistent with the cross-equatorial vorticity transport by the Hadley cell, which brings negative (positive) vorticity into the Northern (Southern) Hemisphere during austral (boreal) summer. This is associated with a southward vorticity transport $(f+\bar{\xi}) \bar{v}<0$ across the equator throughout the year (red line in Fig. 1c), but much stronger during JJA, consistent with the larger displacement of the vorticity equator during this season.

The hemispheric vorticity budget is relevant for understanding the determination of the equatorial wind, as implied by the relations

$$
\begin{aligned}
& \left\langle\xi_{\mathrm{NH}}\right\rangle=\int_{0}^{\pi / 2} \bar{\xi} a \cos \phi d \phi=U_{\mathrm{EQ}}, \\
& \left\langle\xi_{\mathrm{SH}}\right\rangle=\int_{-\pi / 2}^{0} \bar{\xi} a \cos \phi d \phi=-U_{\mathrm{EQ}},
\end{aligned}
$$

where the angle brackets denote hemispheric, areaweighted averages, and $U_{\mathrm{EQ}}$ is the zonal-mean equatorial wind. As noted above, the Hadley cell produces a southward vorticity transport throughout the year. This mean vorticity transport is not balanced by the eddy transport, which is primarily downgradient and hence also southward $\left(\overline{v^{\prime} \xi^{\prime}}<0\right.$; blue line in Fig. 1c). There is thus a net southward advective vorticity transport (thick black line) that can only be balanced by vorticity sources and sinks. One of our goals is to understand the processes that balance this advective vorticity transport.

Consistent with the arguments of Lindzen and Hou (1988), the equatorial winds are easterly during most of the year, with the exception of weak westerlies during boreal winter (Fig. 1d, black line). Nevertheless, the westerly acceleration by the eddy forcing over the summer hemisphere significantly weakens the easterlies that would be observed if angular momentum were conserved. We can get an indication of the impact of the eddy forcing on angular momentum conservation by comparing the observed wind with the wind that would be observed if angular momentum were conserved. The red line in Fig. 1d shows the equatorial wind that would be observed if the Hadley cell conserved the planetary angular momentum at the latitude of maximum zonalmean upwelling, while the blue line shows the same using the mean planetary momentum over the latitudes of zonal-mean tropical upwelling (weighted by the magnitude of this upwelling). It is apparent that the seasonal migration of the upwelling plays an important role for the seasonal cycle of the equatorial wind, even though the Hadley cell is far from angular momentum conserving.

Discussions about the plausibility of terrestrial superrotation usually revolve around the annual-mean equatorial momentum balance. In this context, the analysis of Lee (1999) shows that the main deceleration term on the equator arises from temporal correlations in the seasonal cycles of $\bar{v}$ and $\bar{\xi}$, so it is sometimes argued that "Hadley cell transience" prevents superrotation. However, this annual-mean balance simply reflects the average of the individual seasonal balances (note that $\bar{v}$ and $\bar{\xi}$ have small annual averages and that zonal-wind tendencies are negligible at seasonal time scales), and for each of these, whether superrotation occurs or not depends primarily on the magnitude of the eddy forcing and the latitude and strength of the tropical upwelling. Figure $1 \mathrm{~d}$ shows that terrestrial superrotation does occur during boreal winter, though it is extremely weak, while a much stronger eddy forcing than observed would be required for superrotation during boreal summer due to the large poleward migration of the tropical upwelling associated with the summer Asian monsoon (Bordoni and Schneider 2008; Shaw 2014). The annual-mean equatorial wind is strongly affected by the import of low-angular-momentum air onto the equator during this season. 


\section{Meridional-vertical decomposition of the eddy forcing: Advective and flux forms}

Using the flux form of the momentum equation, Dima et al. (2005) showed that tropical eddy momentum forcing is dominated by the meridional convergence of $\overline{u^{\prime} v^{\prime}}$. However, we show below that the impact of vertical momentum mixing is more important than a cursory reading of their results would suggest. The meridionalvertical decomposition of the tropical eddy forcing is quite different depending on whether the advective or flux form is used. This is demonstrated by Figs. 2 and 3, which show the decomposition of the eddy angular momentum forcing using both formulations:

$$
\begin{aligned}
S= & \left.\frac{1}{\cos \phi} \frac{\partial(\bar{u} \cos \phi)}{\partial t}\right|_{\text {eddy }}=-\frac{1}{a \cos ^{2} \phi} \frac{\partial}{\partial \phi}\left(\overline{u^{\prime} v^{\prime} \cos ^{2} \phi}\right) \\
& -\frac{\partial \overline{u^{\prime} \omega^{\prime}}}{\partial p}=\overline{v^{\prime} \xi^{\prime}}-\overline{\omega^{\prime} \frac{\partial u^{\prime}}{\partial p}} .
\end{aligned}
$$

Based on the flux formulation, the 300-150-hPa eddy acceleration is dominated by the meridional momentum flux convergence (Figs. 2b,c), consistent with the results of Dima et al. (2005). However, the partition is somewhat sensitive to the vertical levels used. As shown in Fig. 3, the vertical momentum flux convergence is weaker than the meridional convergence but not entirely negligible. The vertical convergence partially balances the meridional convergence, strongly peaked at upper levels, and spreads the eddy forcing over a deep layer, especially during DJF. Dima et al. (2005) described a similar momentum flux pattern, which is ubiquitous in comprehensive and idealized models (e.g., Kraucunas and Hartmann 2005; Lutsko 2018; Caballero and Carlson 2018), although the degree of meridionalvertical cancellation differs.

However, the decomposition is quite different when an advective formulation is used [rightmost equality in Eq. (3)]. The meridional advection term is written here as a meridional eddy vorticity flux ${ }^{1}$ so as to emphasize the vorticity dynamics. As noted in the introduction, the classical paradigm for eddy-driven jets is based on the notion that Rossby waves transport easterly momentum as they propagate (Held 1975). In the extratropics, Rossby waves generated by baroclinic instability propagate equatorward

\footnotetext{
${ }^{1}$ The vertical advection term can likewise be written as a vertical flux of $y$ vorticity, but we see little advantage to using a vorticity description in this case because $y$ vorticity is not conserved for 2D motions in the $x-z$ plane. See Harnik et al. (2008) for a description of the coupled gravity-vorticity dynamics.
}

and produce downgradient (negative) vorticity fluxes when they break in the subtropics. These are compensated by positive (upgradient) vorticity fluxes over the source region, which impart a westerly acceleration in the midlatitudes (Vallis 2006). A similar mechanism has been proposed to account for the westerly eddy forcing in the tropics, except that Rossby waves are presumed to be generated by asymmetric tropical heating (see, e.g., Saravanan 1993).

The advective decomposition of the eddy forcing (Figs. 2d,e) is qualitatively consistent with this picture during JJA, which shows a broad $\mathrm{NH}$ region with upgradient vorticity fluxes extending all the way to the equator. In contrast, the $\mathrm{SH}$ region with upgradient vorticity flux is weak and spatially confined during DJF, and it does not reach the equator. During this season, the westerly acceleration at the equator is due instead to vertical momentum advection (Fig. 2e). Similar results are found for both equinox seasons so that, except for the JJA case discussed above, the tropical vorticity fluxes are generally negative (more consistent with a Rossby wave sink than with a Rossby wave source) and the westerly acceleration is imparted by the vertical advection term. Figure 3 shows that this term can also capture the deep vertical structure of the full eddy forcing.

Vertical momentum advection is also associated with vorticity generation via vorticity tilting and vertical vorticity advection (the former being dominant), which accounts for the missing vorticity source noted in the previous section. We can see this by differentiating the vertical advective acceleration meridionally:

$$
\begin{aligned}
\frac{1}{a \cos \phi} \frac{\partial}{\partial \phi}\left(\overline{\omega^{\prime} \frac{\partial u^{\prime}}{\partial p}} \cos \phi\right)= & \left.\frac{1}{a} \overline{\frac{\partial \omega^{\prime}}{\partial \phi} \frac{\partial u^{\prime}}{\partial p}}-\frac{1}{a \cos \phi} \frac{\partial \omega^{\prime}}{\partial \lambda} \frac{\partial v^{\prime}}{\partial p}\right) \\
& -\left(\overline{\omega^{\prime} \frac{\partial \xi^{\prime}}{\partial p}}\right) .
\end{aligned}
$$

The two terms in parentheses on the right-hand side represent the vorticity generation by tilting and vertical advection, respectively. As a result, the net hemispheric vorticity generation by these terms (equal to their cosineweighted integral between the equator and poles) is given by the vertical momentum advection on the equator. This term, indicated by the magenta curve in Fig. 1c, largely closes the hemispheric vorticity balance.

Motivated by the relation between wave propagation and Reynolds stresses [Andrews et al. 1987; note that the baroclinic contribution to the vertical Eliassen-Palm (EP) flux is much smaller than $\overline{u^{\prime} \omega^{\prime}}$ in this region], it is customary to write the eddy momentum forcing in flux form. For instance, the eddy momentum flux pattern described above would be consistent with wave refraction 

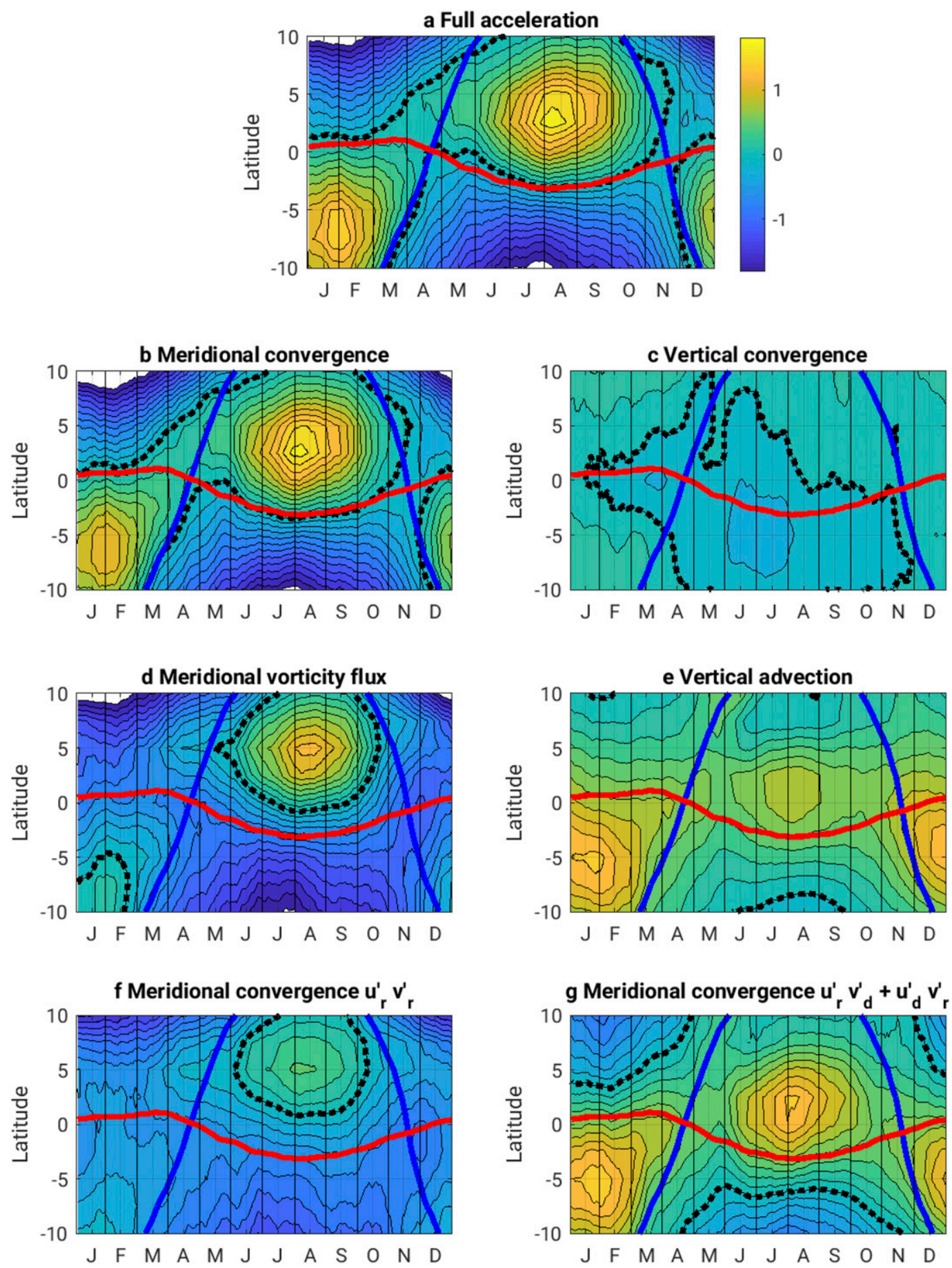

FIG. 2. (a) Full eddy forcing over the 300-150-hPa tropospheric layer as in Fig. 1a and its decomposition into (b) meridional and (c) vertical eddy momentum convergence, and (d) meridional eddy vorticity flux and (e) eddy vertical advection. (f),(g) The decomposition of the meridional eddy momentum convergence in (b) into rotational and divergent contributions, as defined in the text. For clarity, the daily climatologies have been smoothed using a 20-day running mean. The thick black dotted lines mark the zero contours and all panels use the same color bar $\left(\mathrm{m} \mathrm{s}^{-1} \mathrm{day}^{-1}\right)$. 

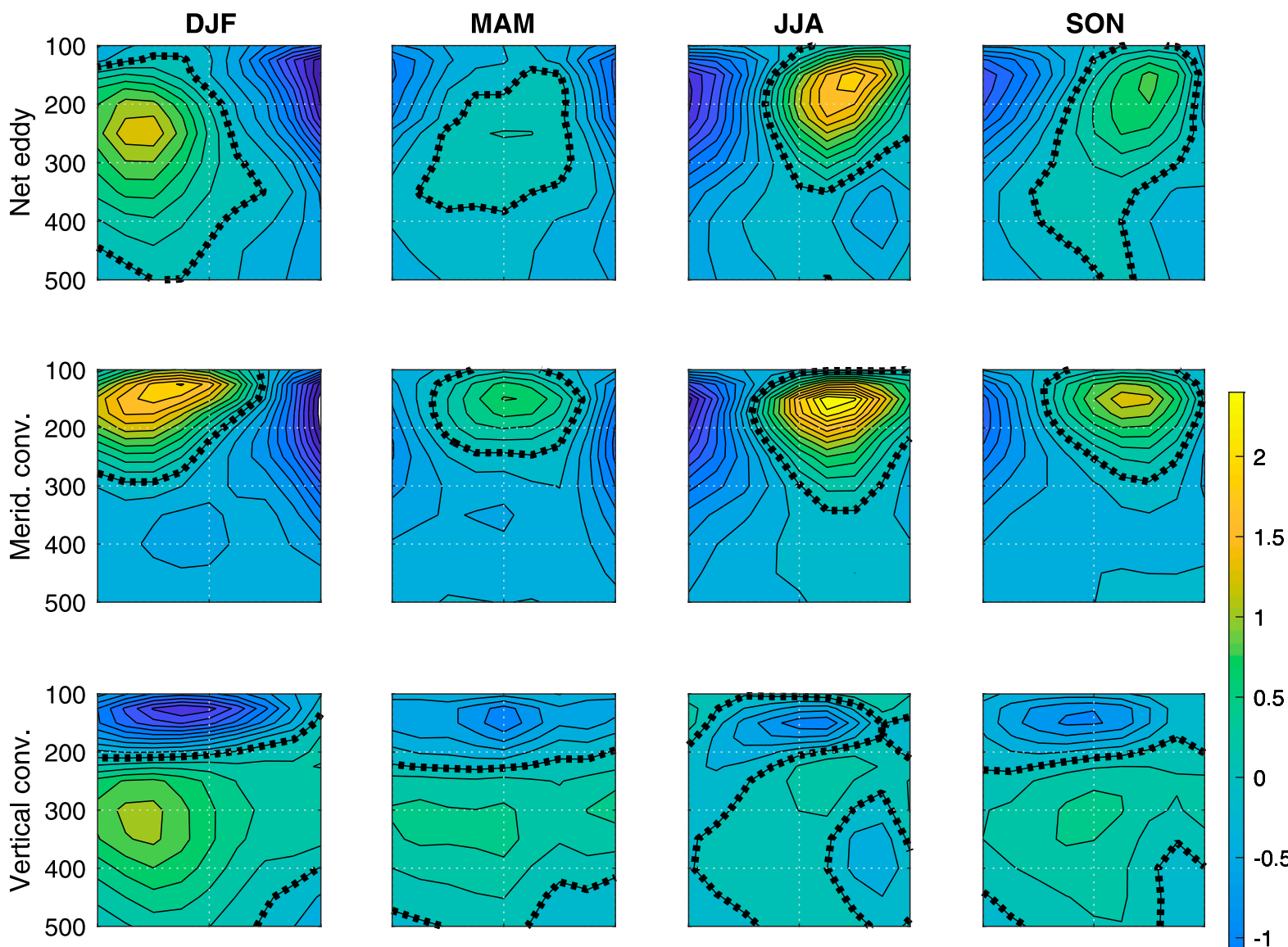

0.5
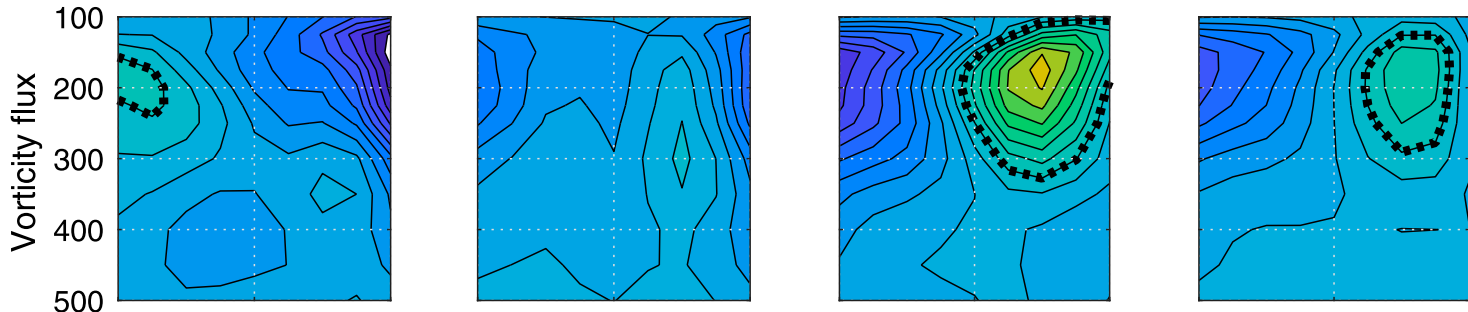

$-2$
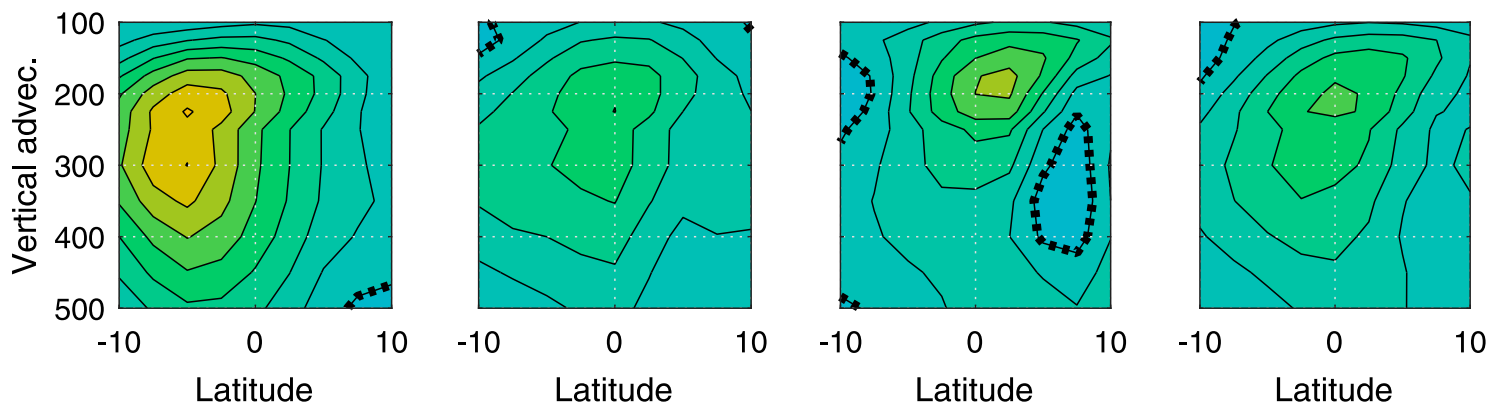

FIG. 3. For the seasons indicated, vertical profiles of (first row) full eddy acceleration and its decomposition into (second row) meridional and (third row) vertical eddy momentum convergence, and (fourth row) meridional eddy vorticity flux and (fifth row) eddy vertical advection. Units are $\mathrm{m} \mathrm{s}^{-1} \mathrm{day}^{-1}$, with the zero contour in thick black dotted line. 
in the $y-z$ plane, as the strong upper-level Hadley flow may favor meridional propagation in the upper troposphere. However, the actual momentum mixing-ultimately related to dissipation-is better represented by the advective term. Zurita-Gotor and Held (2018) discuss a problem in which the convergence of the momentum fluxes fails to induce an Eulerian-mean acceleration in a layer model due to the weakness of the mixing. Our results suggest that although wave propagation is predominantly meridional in the tropical troposphere, the bulk of the mixing actually occurs in the vertical.

The dominance of vertical advection and mixing is connected to the fact that the eddy dissipation driving the mean-flow acceleration in the tropics is diabatic, in contrast with the mechanical dissipation (irreversible Rossby wave breaking) that is key to the spinup of the extratropical jet (Vallis 2006, p. 489). As discussed by Zurita-Gotor and Held (2018), in the presence of a positive-definite vorticity gradient, a westerly eddydriven acceleration requires either a vorticity source or cross-isentropic momentum advection. In the tropics, vertical advection provides a good approximation to cross-isentropic advection: $\overline{\omega^{\prime} \partial u^{\prime} / \partial p} \approx{\dot{Q^{\prime}} \partial u^{\prime} / \partial \Theta}$ due to the near balance between diabatic and adiabatic heating $\dot{Q}^{\prime} \approx \omega^{\prime} \Theta_{p}$. The dominance of eddy vertical advection over the eddy vorticity fluxes (which also have the wrong sign) then suggests that the second route to eddy acceleration of Zurita-Gotor and Held (2018) is more important in the tropical terrestrial atmosphere. In the next section we discuss other differences between tropical and extratropical eddy-driven jets.

\section{Rotational and divergent contributions to the meridional eddy momentum flux convergence}

Eddy-driven jets are maintained against friction by eddy meridional momentum flux convergence. Extratropical eddy-driven jets can be understood using a purely two-dimensional perspective because the propagation and mixing are quasi horizontal (isentropic). In contrast, for tropical eddy-driven jets the momentum mixing occurs predominantly in the vertical. To understand the relation between eddy meridional momentum flux convergence and eddy vertical advection, it is useful to decompose the meridional convergence as follows:

$$
\begin{aligned}
-\frac{1}{a \cos ^{2} \phi} \frac{\partial}{\partial \phi}\left(\overline{u^{\prime} v^{\prime} \cos ^{2} \phi}\right)= & -\overline{\frac{v^{\prime}}{a \cos \phi} \frac{\partial}{\partial \phi}\left(u^{\prime} \cos \phi\right)} \\
& -\overline{\frac{u^{\prime}}{a \cos \phi} \frac{\partial}{\partial \phi}\left(v^{\prime} \cos \phi\right)} \\
& =\overline{v^{\prime} \xi^{\prime}}-\overline{u^{\prime} D^{\prime}},
\end{aligned}
$$

where $D$ is horizontal divergence. The first term, the eddy vorticity flux, dominates in the midlatitudes, where it is responsible for the spinup of the eddydriven jet. However, Fig. $4 \mathrm{~b}$ shows that this term provides a negligible contribution to the annual-mean eddy momentum convergence on the equator and is generally negative over the tropics as discussed above. On the equator, the eddy momentum flux convergence is instead associated with the divergent forcing $\left(-\overline{u^{\prime} D^{\prime}}\right.$; Fig. 4c).

Equation (4) implies that the familiar association between precipitation/upper-level divergence and anomalous upper-level tropical easterlies is associated with momentum transport onto the equator. Figure 5 illustrates the mechanism involved in this meridional momentum transport. A negative $\overline{u^{\prime} D^{\prime}}$ term is associated with meridional mass convergence over longitudinal sectors with westerly wind anomalies and meridional mass divergence over sectors with easterly wind anomalies. As isentropic thickness deepens (shallows) over the former (latter), this configuration leads to an increase in the mass-weighted momentum $\overline{u^{\prime} \sigma^{\prime}}$ via a positive $\overline{u^{\prime} D \sigma^{\prime} / D t}$, where $\sigma$ is isentropic thickness/ density. However, note that by itself, this pattern cannot change the Eulerian-mean momentum $\bar{u}$, only $\overline{u^{\prime} \sigma^{\prime}}$ (Zurita-Gotor and Held 2018). The actual Eulerian acceleration is accomplished by vertical (cross isentropic) momentum advection, which redistributes momentum between the isentropic layers. In pressure coordinates, we expect downward (upward) motion over longitudinal sectors with upperlevel meridional convergence (divergence). Since the former (latter) are associated with westerly (easterly) wind anomalies as noted above, this leads to a westerly acceleration by vertical advection $-\overline{\omega^{\prime} \partial u^{\prime} / \partial p}>0$ whenever the wind has a baroclinic structure or an equivalent barotropic structure with weaker winds near the surface.

We can also decompose the eddy vorticity flux into contributions by the divergent and rotational flow: $\overline{v^{\prime} \xi^{\prime}}=\overline{v_{r}^{\prime} \xi^{\prime}}+\overline{v_{d}^{\prime} \xi^{\prime}}$. (The $\overline{u^{\prime} D}$ term is strongly dominated by the $\overline{u_{r}^{\prime} D}$ contribution, not shown.) Figures $4 \mathrm{~d}$ and $4 \mathrm{e}$ suggest that this decomposition can be useful for separating the fluxes arising from tropicalextratropical interactions from the internal tropical dynamics. The rotational component (Fig. 4d) has a deeper vertical structure and is associated with momentum exchange between tropics and extratropics. Since we can express $\overline{v_{r}^{\prime} \xi^{\prime}} \cos \phi$ as the convergence of a flux,

$$
\overline{v_{r}^{\prime} \xi^{\prime}} \cos \phi=-\frac{1}{a \cos \phi} \frac{\partial}{\partial \phi}\left(\overline{u_{r}^{\prime} v_{r}^{\prime}} \cos ^{2} \phi\right),
$$



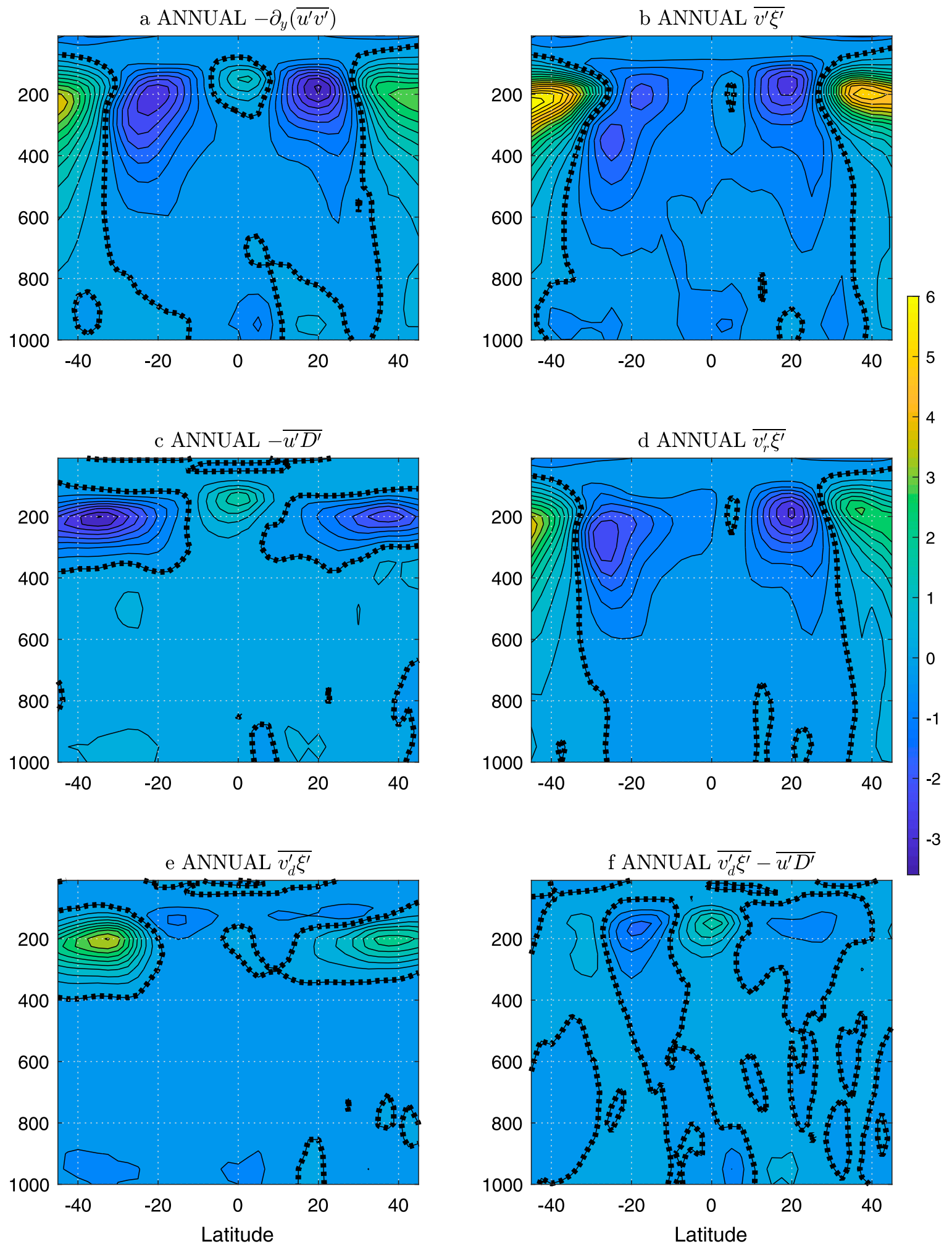

FIG. 4. (a) Annual-mean eddy meridional momentum convergence $\left(\mathrm{m} \mathrm{s}^{-1} \mathrm{day}^{-1}\right)$ and contributions by (b) total vorticity flux, (c) $-\overline{u^{\prime} D^{\prime}}$, (d) rotational vorticity flux, (e) divergent vorticity flux, and (f) total divergent momentum convergence. The thick black dotted lines mark the zero contours. 


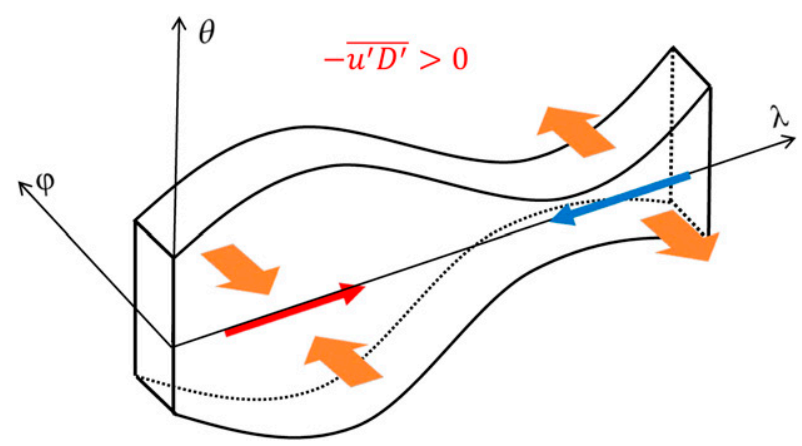

FIG. 5. Sketch illustrating the mechanism of eddy momentum convergence by the divergent circulation (wide orange arrows). When $\overline{u^{\prime} D^{\prime}}<0$, there is meridional mass convergence over longitudinal sectors with eddy westerlies (red arrow) and meridional mass divergence over sectors with eddy easterlies (blue arrow). This increases (decreases) isentropic thickness over the former (latter) longitudes, and leads to an increase in the mass-weighted momentum $\overline{u^{\prime} \sigma^{\prime}}$.

this term integrates globally to zero. In contrast, the divergent eddy vorticity flux $\overline{v_{d}^{\prime} \xi^{\prime}}$ (Fig. 4e) is very top-heavy, though small in the deep tropics. It is positive and large in the subtropical upper troposphere, where it compensates the deceleration by the $\overline{u^{\prime} D^{\prime}}$ term (Fig. 4c). It is easy to show that the sum of these two terms can again be expressed as the convergence of a flux and hence also integrates globally to zero:

$$
\left(\overline{v_{d}^{\prime} \xi^{\prime}}-\overline{u_{r}^{\prime} D^{\prime}}\right) \cos \phi=-\frac{1}{a \cos \phi} \frac{\partial}{\partial \phi}\left[\left(\overline{u_{r}^{\prime} v_{d}^{\prime}}+\overline{u_{d}^{\prime} v_{r}^{\prime}}\right) \cos ^{2} \phi\right] .
$$

As will be shown below, the first of the two components on the right-hand side of Eq. (6) strongly dominates: $\overline{u_{r}^{\prime} v_{d}^{\prime}} \gg \overline{u_{d}^{\prime} v_{r}^{\prime}}$, which attests to the relevance of the longwave approximation in the tropics (note that $u_{r}^{\prime}$ and $v_{d}^{\prime}$ are $y$ derivatives of streamfunction and velocity potential, respectively, while $u_{d}^{\prime}$ and $v_{r}^{\prime}$ are $x$ derivatives, and that $k \ll l$ for the dominant momentum-transporting waves). Our diagnostics show that the meridional con-

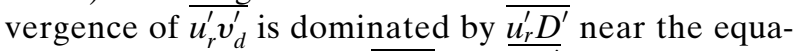
tor, but in the subtropics $\overline{u_{r}^{\prime} D^{\prime}}$ and $\overline{v_{d}^{\prime} \xi^{\prime}}$ are comparable and largely cancel each other, leaving a net momentum divergence as a residue. Given the extent of the cancellation, we speculate that these terms might be associated with a change in the structure of the waves (more divergent in the tropics and more rotational in the subtropics) as they propagate from one region to another, rather than with true wave-mean flow interaction (pseudomomentum forcing), but this question needs to be investigated further.

In the following we will refer to the sums in Eq. (6) as the divergent eddy momentum flux and eddy momentum flux convergence (even though they also involve the rotational flow) to distinguish them from the purely rotational flux and convergence described above. This designation is also motivated by the analysis (to be presented in Part II) that suggests that the rotational flow $u_{r}^{\prime}$ is to a large extent forced by the divergent motions in the tropics. Figure $4 \mathrm{f}$ shows that the divergent momentum flux so defined is associated with momentum transport from the subtropics to the equatorial region and is weak at high latitudes. Finally, we note that there is also a purely divergent contribution to the eddy momentum convergence:

$$
-\overline{u_{d}^{\prime} D^{\prime}} \cos \phi=-\frac{1}{a \cos \phi} \frac{\partial}{\partial \phi}\left(\overline{u_{d}^{\prime} v_{d}^{\prime}} \cos ^{2} \phi\right) .
$$

This contribution is much smaller than the terms included in Fig. 4 (not shown) and will not be discussed further.

To conclude, Fig. 6 shows the partition of the meridional momentum flux convergence for the two solstice seasons, with results similar to those presented above. As for the annual mean, the equatorial convergence is dominated by the divergent momentum flux in both seasons, though the rotational component has a small contribution during JJA. The role of this term is nevertheless much less important than suggested by the seasonal cycle of the full eddy vorticity flux (the dominant JJA forcing; cf. Fig. 2d), since a significant fraction of this vorticity flux is in fact associated with $\overline{v_{d}^{\prime} \xi^{\prime}}$. This is demonstrated by the bottom two panels of Fig. 2, which show how the annual cycle of the eddy meridional momentum convergence is partitioned into its rotational and divergent components. We can see that the divergent momentum flux is the leading acceleration term, even during JJA, by virtue of the $\overline{v_{d}^{\prime} \xi^{\prime}}$ contribution. We speculate that the enhanced role of the eddy vorticity flux (both rotational and divergent) during JJA compared to DJF might be due to enhanced vorticity generation in the tropics during boreal summer in association with the Tibetan anticyclone.

\section{Analysis of the divergent momentum flux}

We have shown above that eddy momentum flux convergence in the tropics is dominated by the divergent momentum flux. However, the same is not necessarily true for the momentum flux, because the above diagnostics could also be compatible with a cross-equatorial rotational momentum flux connecting the two subtropical hemispheres, with weak convergence in the deep tropics. Motivated by recent findings on such an interhemispheric teleconnection ( $\mathrm{Ji}$ et al. 2014; Li et al. 2015), 

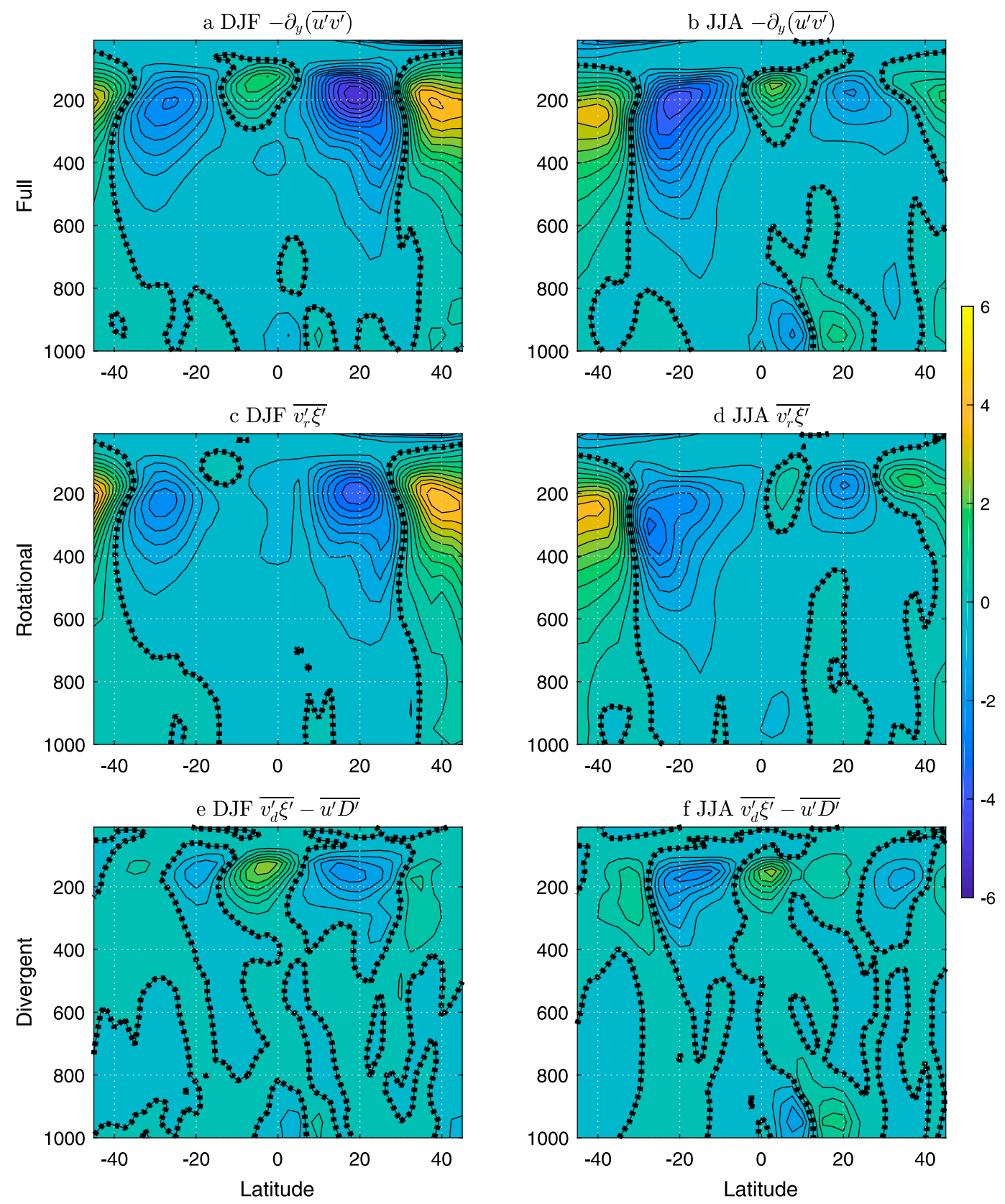

FIG. 6. As in Figs. 4a, 4d, and 4f, but for (a),(c),(e) DJF and (b),(d),(f) JJA.

Figs. 7 and 8 show the partitioning of the tropical momentum flux into its rotational and divergent components during DJF and JJA, respectively.

During both solstice seasons, wave propagation is directed from the summer to the winter hemisphere (Figs. 7a, 8a). The cross-equatorial rotational (blue) and divergent (red) momentum fluxes are of the same sign, but the latter are more than twice as large on the equator. We can also see that the divergent eddy momentum flux is strongly dominated by the $\overline{u_{r}^{\prime} v_{d}^{\prime}}$ component (dashed red line), as anticipated above. The divergent eddy momentum flux is associated with the region of strong meridional momentum convergence adjacent to the equator in the summer hemisphere (in JJA, a second peak of momentum flux convergence at higher northern latitudes is also apparent).

In contrast, the rotational momentum flux is associated in both hemispheres and seasons with waves generated at higher latitudes. During DJF the rotational 

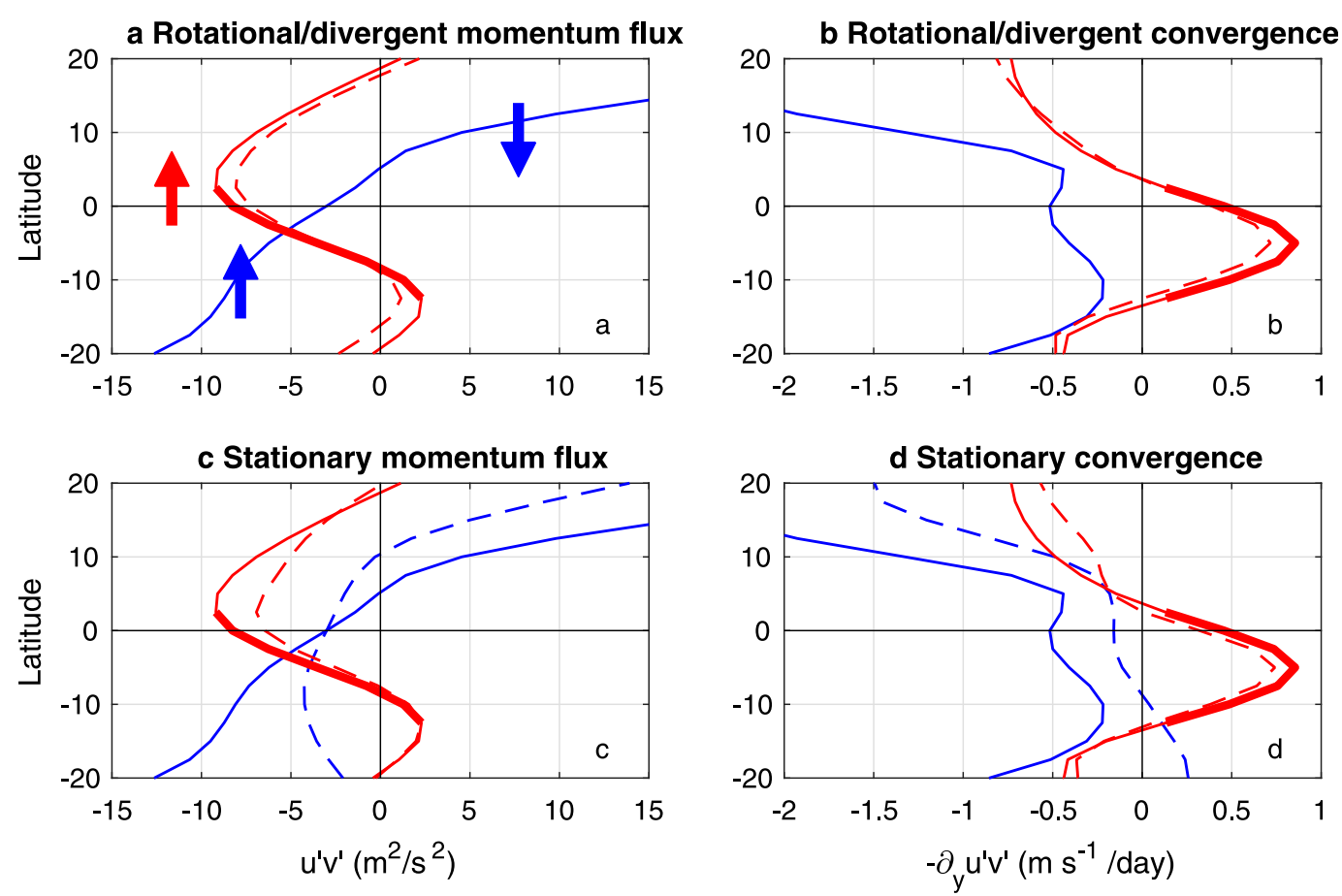

FIG. 7. During DJF, (a) rotational (blue) and divergent (red; the $\overline{u_{r}^{\prime} v_{d}^{\prime}}$ contribution is shown with a dashed line) eddy momentum fluxes and (b) their convergence. The arrows in (a) show the direction of propagation as depicted by the associated EP fluxes and latitudes with positive momentum convergence are emphasized with a thick line in both panels. (c),(d) Contribution of the stationary momentum fluxes (dashed) to the full eddy momentum flux and convergence (solid). All variables are averaged over the $150-300-\mathrm{hPa}$ layer.

momentum flux diverges throughout the tropics but during JJA there is a narrow band of (weak) eddy momentum flux convergence slightly to the north of the equator. In the deep tropics the rotational momentum flux is larger in the summer than in the winter hemisphere but the contrary is true poleward of about $15^{\circ}$. The latter is consistent with the seasonal cycle in extratropical eddy generation, which gives rise to stronger Rossby wave propagation into the winter subtropics. However, because the rotational wave flux does not penetrate deep into the tropics in this hemisphere, close to the equator propagation from the summer hemisphere dominates. This may reflect the asymmetry in propagation introduced by the Hadley cell flow (Schneider and Watterson 1984; Li et al. 2015), or it could be due to the stronger Rossby wave forcing in the summer tropics.

The bottom panels of Figs. 7 and 8 show with dashed lines the rotational and divergent momentum fluxes and flux convergence due to the stationary eddies alone. Dima et al. (2005) already noted the dominance of the stationary momentum flux in the tropics but it is interesting to perform the analysis for the rotational and divergent components separately. As expected, the stationary rotational momentum flux is larger in the
Northern Hemisphere than in the Southern Hemisphere, particularly during DJF, when extratropical planetary waves propagating into the tropics are known to force the Hadley cell (Zurita-Gotor and Álvarez-Zapatero 2018). In contrast, the stationary divergent momentum flux has a very similar structure to the full divergent momentum flux: it peaks near the equator in the summer hemisphere and decays away rapidly on the winter side of the equator. It is apparent that the stationary wave component dominates the divergent momentum flux, especially during DJF.

Figures $9 \mathrm{a}$ and $9 \mathrm{~b}$ show the climatological-mean upper-level eddy divergence $D^{\prime}$ and $\left(u_{r}^{\prime} v_{d}^{\prime}\right)$ eddy wind vectors that produce the observed stationary divergent eddy momentum flux during DJF and JJA, respectively. ${ }^{2}$ During DJF, we observe northward cross-equatorial flow over the Indian Ocean-western Pacific sector, a region with climatological-mean eddy easterlies, and southward cross-equatorial flow over the eastern Pacific-tropical

\footnotetext{
${ }^{2}$ To keep the notation simple, we do not use any special symbol to denote stationary wave components. Eddy (primed) anomalies will represent climatological-mean values for the remainder of this section.
} 

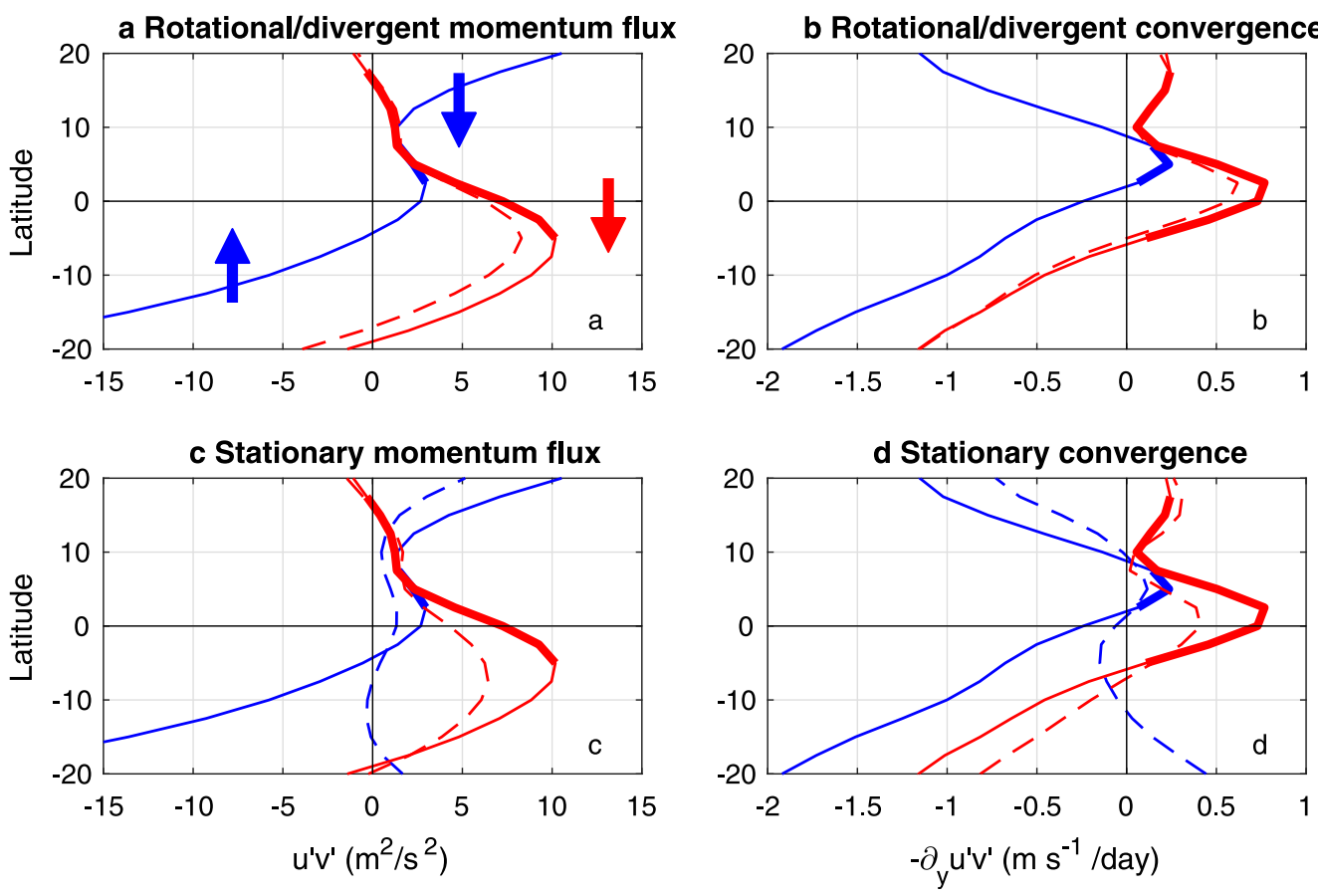

FIG. 8. As in Fig. 7, but for JJA.

Atlantic sector, a region with climatological-mean eddy westerlies. The $v_{d}^{\prime}$ convergence gives rise to eddy momentum flux convergence and a westerly acceleration through the $\overline{u^{\prime} D^{\prime}}$ term, as discussed in the previous section. The reverse $v_{d}^{\prime}$ pattern is found during JJA. It is also apparent from Figs. 9a and 9b that the stationary momentum flux is dominated by long zonal waves, especially during JJA. Figure $9 \mathrm{~d}$ shows the seasonal cycle of the stationary momentum flux due to zonal wavenumber $k=1$ alone, which should be compared to the full stationary momentum flux in Fig. 9c. The $k=1$ contribution accounts for over $70 \%$ $(90 \%)$ of the $\overline{u_{r}^{\prime} v_{d}^{\prime}}$ amplitude during boreal winter (summer), with the remaining DJF momentum flux almost entirely due to zonal waves $k=2$ and $k=3$ (not shown).

Figure 10 describes the zonal structure contributing to the stationary momentum transport in more detail. The top two panels show the seasonal cycles of the uppertroposphere, monthly-mean $u_{r}^{\prime}$ and $v_{d}^{\prime}$, latitudinally averaged within $10^{\circ}$ of the equator, for all waves (left) and for $k=1$ alone (right). The rotational $u_{r}^{\prime}$ maintains a roughly constant phase throughout the year, with eddy westerlies in the Western Hemisphere and eddy easterlies in the Eastern Hemisphere. However, its amplitude is significantly stronger during northern winter than during northern summer. This is not just due to the presence of higher zonal wavenumbers during DJF: even for $k=1$ the $u_{r}^{\prime}$ amplitude is $50 \%$ larger during this season than during JJA. In contrast, the divergent $v_{d}^{\prime}$ has a similar amplitude during both solstice seasons but its phase reverses throughout the seasonal cycle as the ITCZ migrates into the summer hemisphere. Year-round, the strongest convection and cross-equatorial flow is found to the west of the date line over the warm pool region, giving rise to southerly (northerly) eddy meridional winds over this region during DJF (JJA). This seasonal reversal in $v_{d}^{\prime}$ as $u_{r}^{\prime}$ keeps a constant phase explains the sign change in the eddy momentum flux (Fig. 10, bottom right).

Although one can capture the latitudinal structure and much of the amplitude of the zonal-mean $\overline{u_{r}^{\prime} v_{d}^{\prime}}$ flux at all seasons using zonal wave $k=1$ alone, higher harmonics lead to the localization of this flux over some longitudinal sectors (Fig. 10, bottom left). During DJF, the bulk of the southward eddy momentum transport is found over the tropical eastern Pacific, with a secondary peak over the tropical eastern Atlantic. In contrast, during JJA the northward eddy momentum transport is strongly localized over the Indian Ocean. The former is consistent with the existence of a westerly duct favoring cross-equatorial propagation over the eastern Pacific (Hoskins and Ambrizzi 1993). Although zonal winds are easterly at all longitudes during JJA, it has been argued by Schneider and Watterson (1984) and Li et al. (2015) that the Hadley cell flow allows along-stream Rossby wave propagation through easterly winds. However, all these ideas about Rossby wave propagation come 
a DJF Upper-level eddy divergence and $\left(u^{\prime}{ }_{r}, v{ }_{d}\right)$ wind vectors
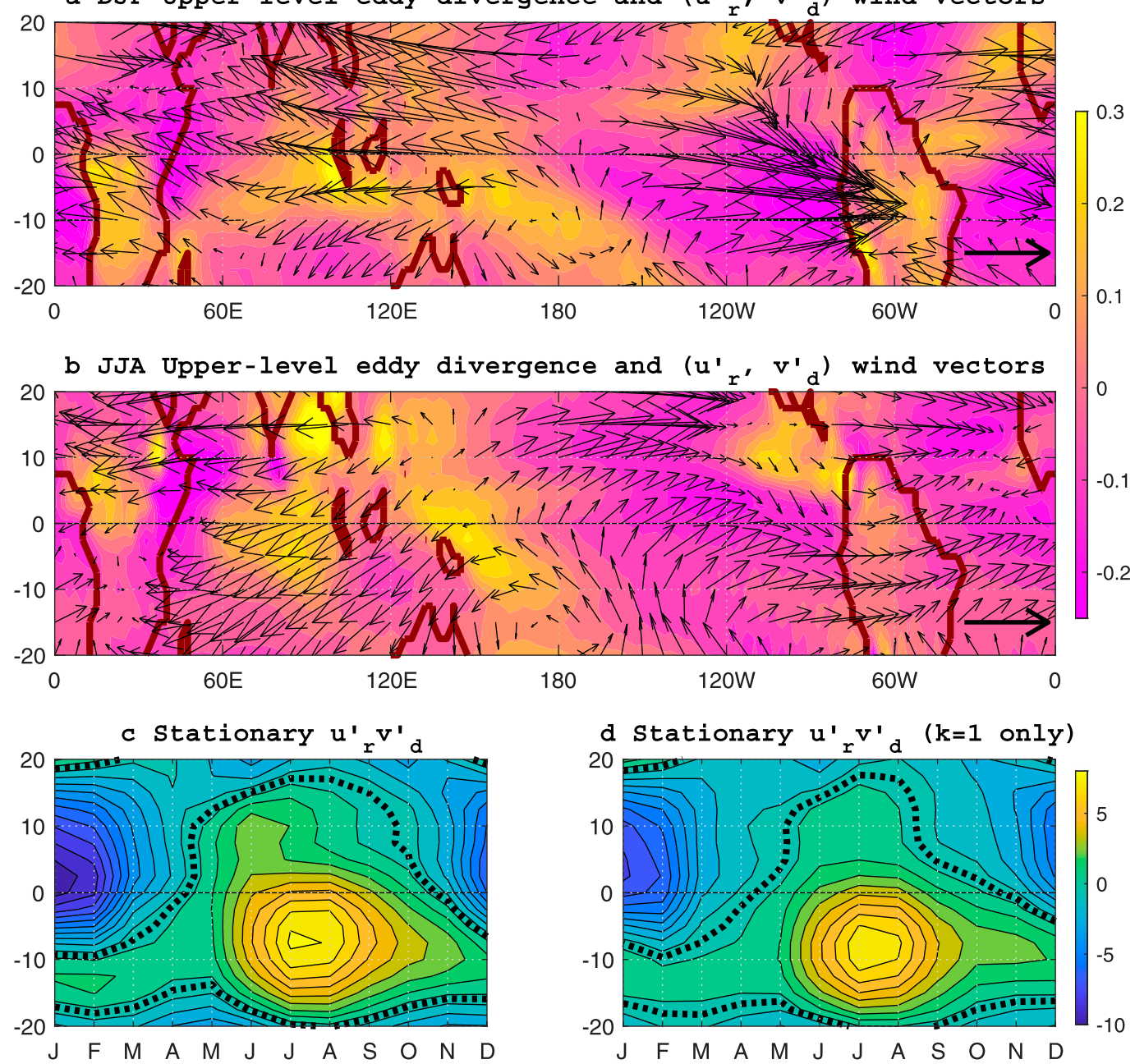

FIG. 9. (a) DJF climatology of upper-level (150-300-hPa average) eddy divergence (shading; day $\left.{ }^{-1}\right)$ and $\left(u_{r}^{\prime}, v_{d}^{\prime}\right)$ wind vectors (reference vector: $10 \mathrm{~m} \mathrm{~s}^{-1}$ ). (b) As in (a), but for the JJA climatology. (c) Seasonal cycle of stationary $\overline{u_{r}^{\prime} v_{d}^{\prime}}$, with the zero contour shown in thick black dotted line. (d) As in (c), but for the $k=1$ contribution alone.

from barotropic theory and only concern the rotational momentum fluxes, while the bulk of the momentum transport is divergent. We discuss the generalization of these ideas in Part II.

\section{MJO eddy momentum transport}

We showed above that the stationary wave contribution dominates the divergent eddy momentum flux in the tropics (consistent with Dima et al. 2005), with some seasonal differences. The transient contribution is negligible during DJF (Fig. 7d) but nearly as large as the stationary contribution during JJA (Fig. 8d; see also Figs. 1a,b). Lee (1999) used spectral analysis (in annual data) to show that the transient eddy momentum flux is dominated by interannual variability in the tropics, with the MJO playing a much smaller role. However, the MJO impact on momentum transport may be enhanced in a warmer climate and potentially lead to superrotation (Lee 1999; Caballero and Huber 2010). Motivated by this, we investigate in this section the dynamics of the MJO momentum transport by means of regression analysis using the diagnostics of Adames and Wallace (2014).

The top three panels of Fig. 11 show regressions of upper-level divergence (shading) and $\left(u_{r}^{\prime}, v_{d}^{\prime}\right)$ wind vectors on the standardized extended winter (NDJFM) MJO time series for equispaced phases of an MJO half period. The same patterns with reversed signs would be observed during the second half period, and only anomalies significant at the $95 \%$ level are shown. The sequence shown corresponds to the most active and slowest phase of MJO progression, during which the longitude of maximum equatorial $\Delta \chi_{150-850}(\mathrm{red}$ 

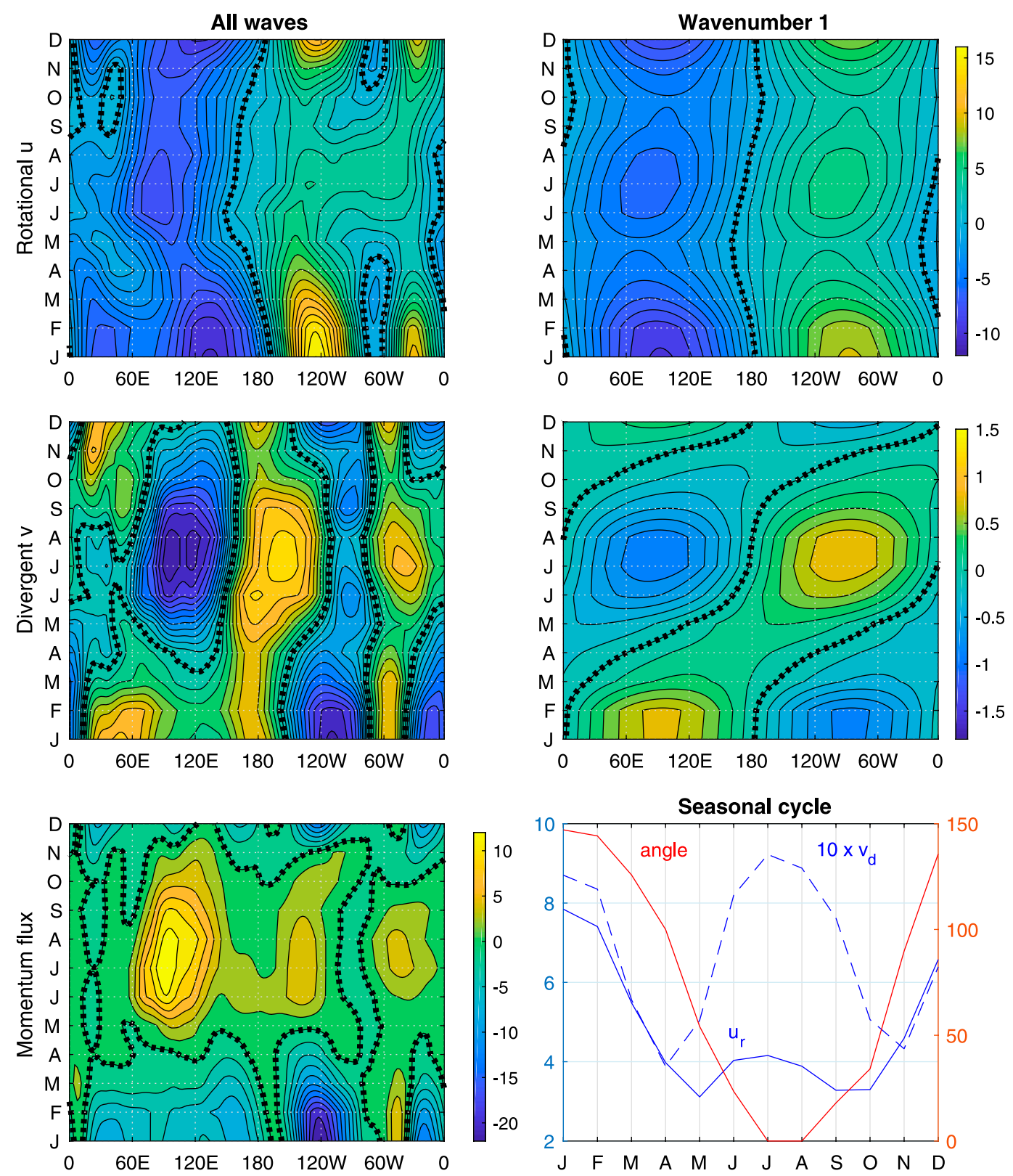

Fig. 10. (left) Annual cycle of the monthly mean eddy wind components (top) $u_{r}^{\prime}$ and (middle) $v_{d}^{\prime}$, averaged between 150 and $300 \mathrm{hPa}$ and between $10^{\circ} \mathrm{S}$ and $10^{\circ} \mathrm{N}$, and (bottom) their product, the stationary momentum flux $u_{r}^{\prime} v_{d}^{\prime}$. (right) The $k=1$ contributions to the (top) $u_{r}^{\prime}$ and (middle) $v_{d}^{\prime}$ fields, and (bottom) the seasonal cycles of the $u_{r}^{\prime}$ and $v_{d}^{\prime}$ norms (RMS amplitude for all waves) and their relative phase, defined as $\theta=\cos ^{-1}\left(\overline{u_{r}^{\prime} v_{d}^{\prime}}|| u_{r}^{\prime}|| v_{d}^{\prime} \mid\right)$.

marker in Fig. 11) moves from the Maritime Continent to the eastern Pacific. Inspection of these maps shows predominance of easterly (westerly) zonal winds over regions of upper-level $v_{d}^{\prime}$ divergence (convergence). Upper-level divergence dominates for the first two maps, while convergence is also apparent over the Indian Ocean in the third map, by which time the MJO peak has moved into the eastern Pacific and convection is suppressed over the warm pool. This pattern is associated with equatorial momentum flux convergence $\overline{u^{\prime} D^{\prime}}<0$ through the mechanism described in section 5 and with southward cross-equatorial momentum flux due to the meridional asymmetry in $v_{d}^{\prime}$, as upper-level divergence peaks in the SH during this season.

Figure 12a shows the evolution of the NDJFM MJOdriven meridional eddy momentum flux through an MJO period, calculated by averaging zonally the products of the zonal and meridional wind anomalies for 

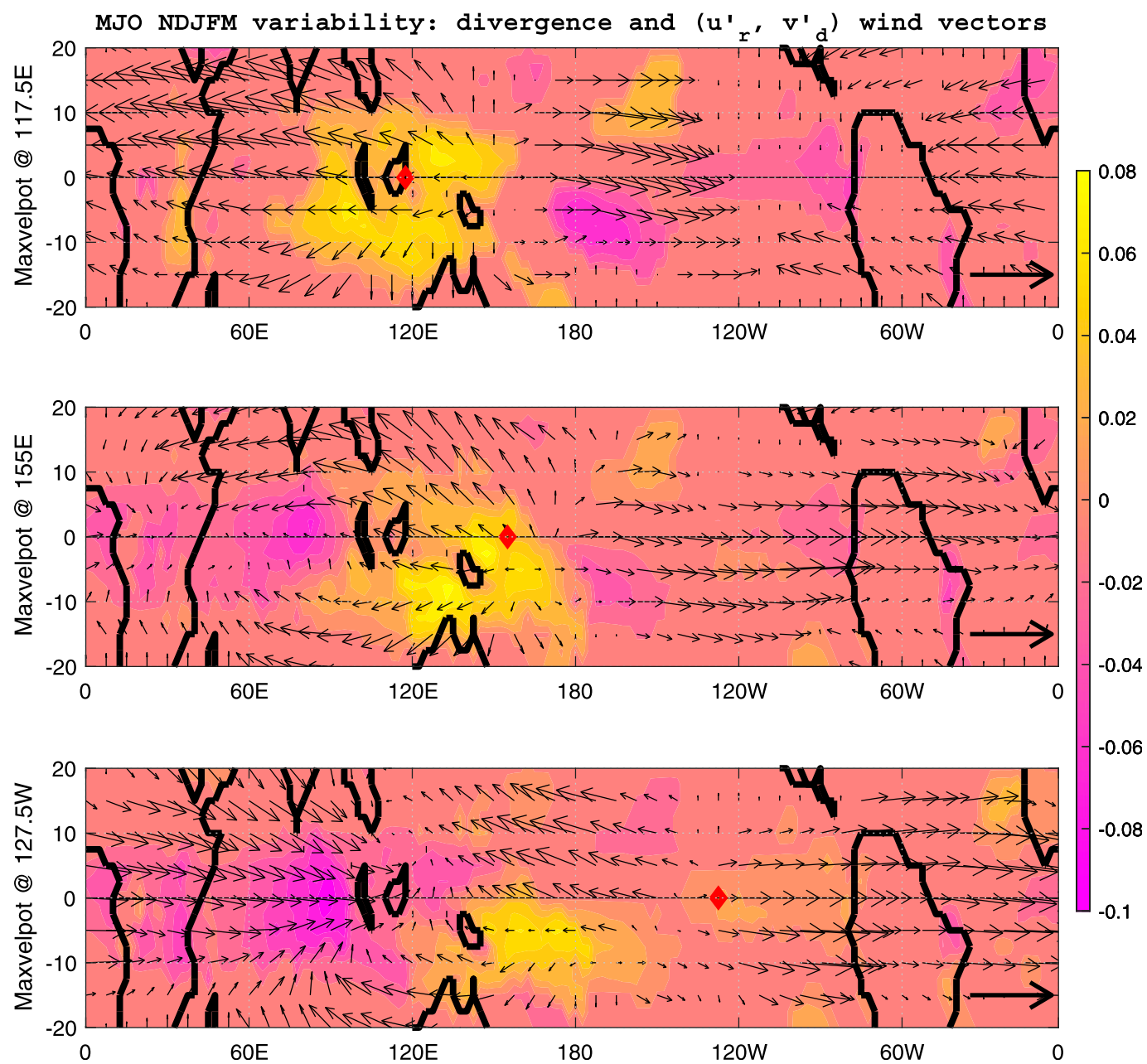

FIG. 11. Regression of upper-level (150-300-hPa average) divergence (shading; day ${ }^{-1}$ ) and $\left(u_{r}^{\prime}, v_{d}^{\prime}\right)$ wind vectors (reference vector: $5 \mathrm{~m} \mathrm{~s}^{-1}$ ) on the standardized MJO index of Adames and Wallace (2014) during NDJFM for three equally spaced phases covering a half period of the oscillation. The MJO phase (longitude of maximum velocity potential difference) is indicated by the red marker at the equator, and only anomalies significant at the $95 \%$ level are shown.

the different MJO phases (phases are expressed here in terms of the longitude of maximum $\Delta \chi$ ). The eddy momentum transport is strongest during the peak MJO phases, when convection is either strongest or most strongly suppressed over the Maritime Continent. This momentum flux is dominated by the divergent component (Fig. 12b), consistent with the mechanism described above. However, although the MJO-related momentum flux has the same sign and structure as the full momentum flux, it is an order of magnitude smaller than the stationary momentum flux in Fig. 9c, consistent with the diagnostics of the previous section and the results of Lee (1999). The same is true during the extended northern summer (JJAS; Figs. 12c,d) even though the transient contribution to the divergent momentum flux is more important during this season.
The weakness of the MJO momentum transport is simply due to the small magnitude of the MJO anomalous divergence and wind vectors compared to those in the climatological-mean stationary waves (note the different scales in Figs. 9 and 11).

On the other hand, Grise and Thompson (2012) have shown that tropical eddy momentum transport is significantly modulated by the MJO, with the regressed eddy momentum flux exceeding $3 \mathrm{~m}^{2} \mathrm{~s}^{-2}$ (cf. their Fig. 9). Although these anomalies are larger than the MJO fluxes in Figs. 12a and 12b, they produce no net momentum transport when averaged over an MJO period. This is illustrated in Fig. 12e, which shows the regression of the daily eddy momentum flux on the MJO as a function of phase for the NDJFM season. We can see that tropical eddy momentum transport is enhanced when the $\Delta \chi$ peak 

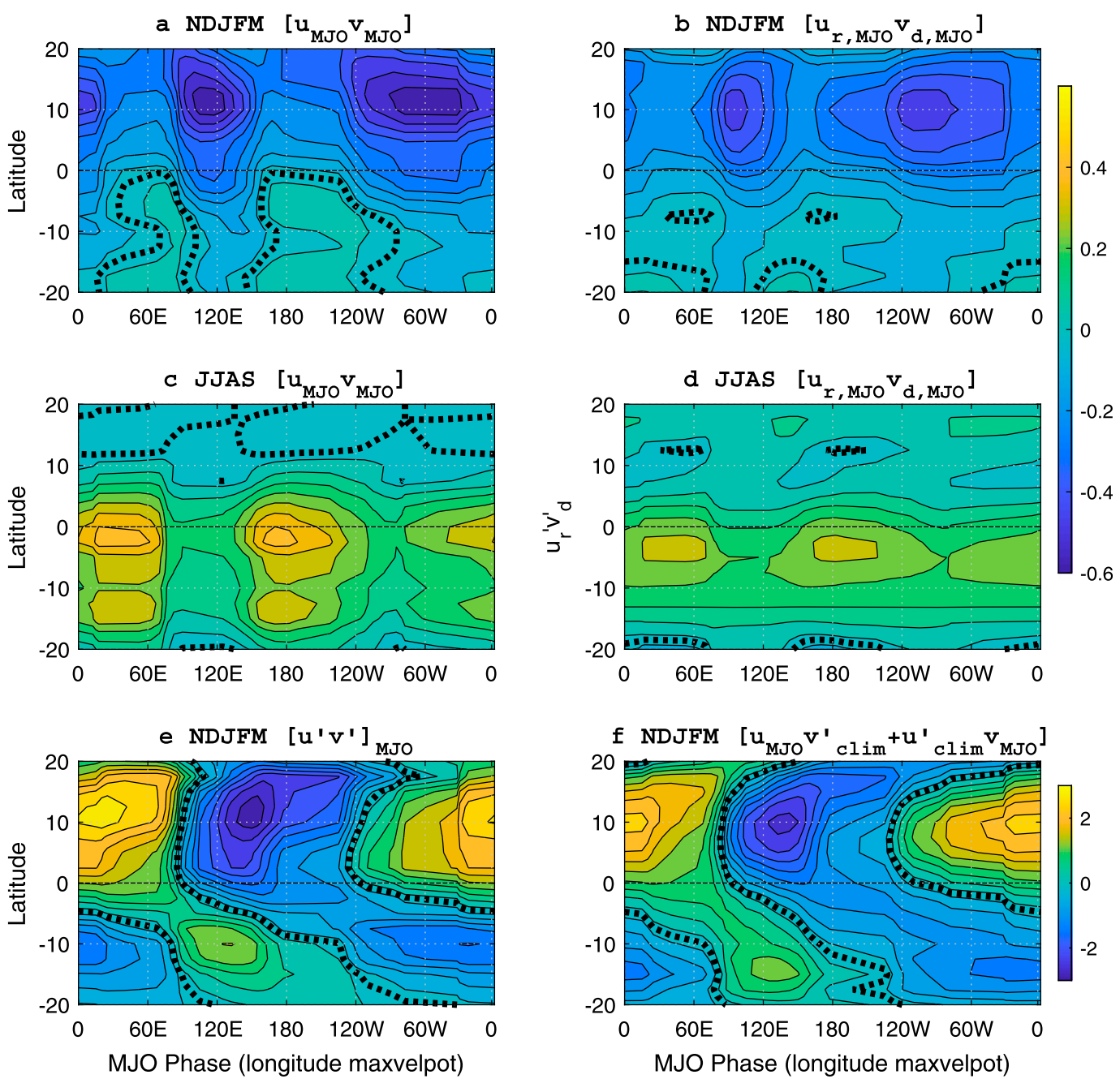

FIG. 12. (a) MJO momentum flux (zonal mean of the product of the regressed MJO velocity anomalies) as a function of the MJO phase, expressed in terms of the longitude of maximum velocity potential. (b) As in (a), but for the divergent contribution $\overline{u_{r}^{\prime} v_{d}^{\prime}}$. (c) As in (a), but for JJAS. (d) As in (b), but for JJAS. (e) Regression of the daily eddy momentum flux $\overline{u^{\prime} v^{\prime}}$ on the MJO index during NDJFM. (f) Linear superposition between the MJO anomaly and the climatological stationary wave: $\overline{u_{\text {clim }}^{\prime} v_{\mathrm{MJO}}}+\overline{u_{\mathrm{MJO}} v_{\mathrm{clim}}^{\prime}}$.

is located over the equatorial Pacific and Maritime Continent, and suppressed at other phases of the MJO period. These correspond to phases of constructive and destructive superposition between the MJO anomaly and the climatological-mean stationary waves, as demonstrated by the good agreement between Fig. 12e and the simple linearization $\overline{u_{\text {clim }}^{\prime} v_{\mathrm{MJO}}}+\overline{u_{\mathrm{MJO}} v_{\text {clim }}^{\prime}}$ in Fig. $12 \mathrm{f}$ ( $u_{\text {clim }}^{\prime}$ and $v_{\text {clim }}^{\prime}$ are the climatological eddy winds and $u_{\mathrm{MJO}}$ and $v_{\mathrm{MJO}}$ the regressed MJO anomalies) and consistent with the arguments of Grise and Thompson (2012). If the MJO anomalies are $O(\varepsilon)$ smaller than the climatological-mean anomalies, the linear interference effect will produce changes in the momentum flux of the same order through an MJO period, with no net rectified transport. The actual MJO momentum transport averaged over the MJO period will be smaller, $O\left(\varepsilon^{2}\right)$. The results presented in this section point to $\varepsilon$ values around 0.3 .

\section{Summary and concluding remarks}

In this work, we have analyzed the role played by the divergent circulation for tropical eddy momentum transport. Although the importance of the divergent flow is of course well recognized in the tropics, the implications for meridional eddy momentum transport appear not to have been investigated before. Since the role of tropical eddies in the determination of the climatological-mean tropical winds has received little attention until relatively recently (Lee 1999; 
Dima et al. 2005), the dynamics of tropical eddy momentum transport has not been studied in as much depth as other aspects of the tropical circulation.

The important role of the divergent momentum transport is a consequence of the diabatic character of the tropical circulation. Although wave propagation is predominantly meridional (implying dominance of meridional eddy momentum fluxes), the bulk of the forcing/ dissipation is associated with cross-isentropic momentum transport and vertical mixing. This entails a very different mechanism of meridional eddy momentum flux convergence from the extratropical jet, associated with mass convergence over sectors with anomalous westerlies and mass divergence over sectors with anomalous easterlies, rather than with vorticity fluxes. This mechanism is similar to that described by ZuritaGotor and Held (2018) for coupled Kelvin-Rossby instability, except that the cross-isentropic momentum transport is presumably due to convective heating in the terrestrial atmosphere.

The dominant component of the eddy momentum flux in the tropics is associated with correlations between the rotational zonal velocity and the divergent meridional velocity: $\overline{u_{r}^{\prime} v_{d}^{\prime}}$. This is the case both for the stationary wave momentum transport and for the MJO momentum flux, though the former dominates due to the larger amplitude of the stationary wave. The symmetric term $\overline{u_{d}^{\prime} v_{r}^{\prime}}$ is significantly smaller, consistent with the large zonal scales of the momentum-transporting waves. This implies that the Walker cell, associated with the divergent zonal flow $u_{d}^{\prime}$, is not important for tropical momentum transport. Instead, our results suggest that tropical eddy momentum transport arises primarily from correlations between zonal anomalies in the meridional overturning/Hadley cell flow and the uppertroposphere Rossby gyres. We can understand the seasonal reversal in the eddy momentum flux by noting that the Rossby gyres keep a roughly constant phase throughout the year, whereas the zonally varying meridional overturning changes sign from winter to summer as the strongest convection and cross-equatorial flow are found west of the date line during both solstice seasons. The stationary momentum transport is dominated by the gravest zonal wave $k=1$. This description of tropical eddy momentum transport is consistent with the findings of Grise and Thompson (2012) that eddy momentum flux variability in the tropics is driven by changes in the amplitude of the equatorial stationary waves. In particular, the reduction in the eddy momentum flux during the warm ENSO phase (Adames and Wallace 2017) can be understood by noting that the weakening of the equatorial stationary wave is associated with a more symmetric Hadley cell.
Because the tropical momentum flux involves both rotational and divergent anomalies, one cannot infer the sign of the eddy momentum flux from the meridional tilt of the isohypses alone- the full velocity vectors need to be considered. The sign of the divergent eddy momentum flux is instead determined by the relative phases of the divergent and rotational anomalies, which requires some coupling between them. We can envision two simple, idealized models for this coupling. First, one could have nearly balanced, remotely forced rotational waves that develop a secondary divergent circulation through adjustment to balance as they propagate into the tropics, similar to the ageostrophic circulation of extratropical Rossby waves. This could be a reasonable model for weakly convecting atmospheres. Alternatively (and more plausibly for the strongly convecting Earth), we might envision a diabatically forced divergent circulation generating Rossby waves through vortex stretching and/or meridional advection by the divergent component of the flow (Sardeshmukh and Hoskins 1988).

The key question in this latter scenario is what determines the phase relation between the divergent forcing and the rotational response. Assuming that the forced rotational flow will adjust to balance the vorticity forcing by the divergent flow, the answer will depend on the closure of the vorticity balance. For instance, consider the simple thought scenario of a cross-equatorial southward Hadley circulation with uniform meridional velocity $V$ at upper levels, that is locally enhanced by anomalous southward flow $v_{d}^{\prime}$ centered at and symmetric about some longitude $x_{0}$ (Fig. 13a). This anomalous meridional flow will produce a positive eddy vorticity forcing $-v_{d}^{\prime} \beta>0$ in both hemispheres, which must be balanced by the rotational vorticity tendency. If we assume that this tendency is dominated by the beta term, $D_{r}(f+\xi) / D t \approx v_{r}^{\prime} \beta$, then we must have $v_{r}^{\prime} \approx-v_{d}^{\prime}$ to balance the vorticity source. Thus, $v_{r}^{\prime}$ will also be symmetric about the longitude $x_{0}$, while $\psi^{\prime}$ and $u_{r}^{\prime}$ will be antisymmetric, which implies a zero divergent momentum flux $\overline{u_{r}^{\prime} v_{d}^{\prime}}=0$ (alternatively, $\overline{u_{r}^{\prime} v_{d}^{\prime}}=-\overline{u_{r}^{\prime} v_{r}^{\prime}}=0$ since the streamlines can have no meridional tilt by symmetry). The induced rotational circulation is depicted schematically in Fig. 13b.

On the other hand, if we assume that $V$ is so strong that the vorticity tendency is dominated by meridional vorticity advection by the Hadley flow and the beta term is negligible, $D_{r}(f+\xi) / D t \approx V d \xi^{\prime} / d y$, then the rotational response will consist of a dipolar vorticity pattern with negative (positive) anomalous vorticity north (south) of the equator (Fig. 13c). This will be associated with equatorial westward flow at longitude $x_{0}$ by the twin anticyclones and hence with a positive eddy momentum flux $\overline{u_{r}^{\prime} v_{d}^{\prime}}>0$. More generally, when both terms are included these arguments suggest that the eddy momentum flux 


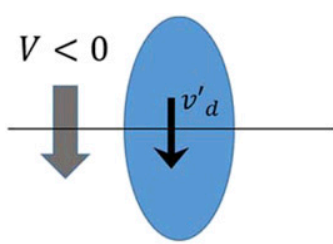

a

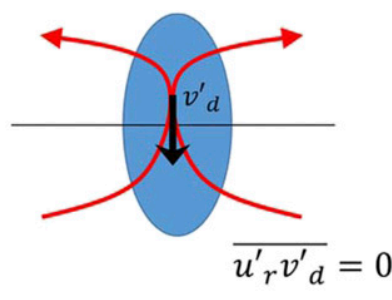

b

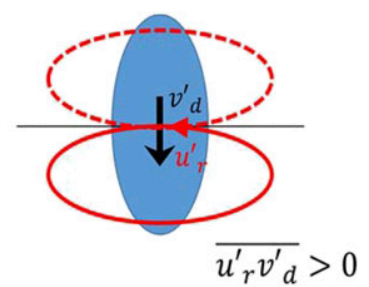

C

FIG. 13. (a) Sketch illustrating two alternative closures of the eddy vorticity balance when the zonal-mean Hadley circulation $V$ is locally enhanced over some longitudes by the anomalous flow $v_{d}^{\prime}$. The positive vorticity forcing $-v_{d}^{\prime} \beta$ over the blue oval is balanced by (b) the beta term $v_{r}^{\prime} \beta$ and (c) the meridional vorticity advection by the Hadley cell $V d \xi^{\prime} / d y$. In (b) the $u_{r}^{\prime}$ and $v_{d}^{\prime}$ anomalies are in quadrature and $\overline{u_{r}^{\prime} v_{d}^{\prime}}=0$. In (c), they are in phase and there is a positive eddy momentum flux: $\frac{u_{r}^{\prime} v_{d}^{\prime}}{>}>0$.

should increase as the Hadley flow $V$ strengthens (of course, other terms may also be important). This thought scenario is interesting because Kraucunas and Hartmann (2007) have found in idealized shallow-water simulations that the cross-equatorial eddy momentum flux is significantly enhanced in the presence of a mean Hadley cell. While Kraucunas and Hartmann (2007) attribute this enhancement to the impact of the mean meridional flow on Rossby wave propagation predicted by nondivergent theory (Schneider and Watterson 1984), we have shown in this paper that the eddy momentum flux is dominated by the divergent component in observations. We will analyze in more detail in Part II the dynamical determinants of the observed $u_{r}^{\prime}-v_{d}^{\prime}$ correlation.

We conclude with a caveat. As most previous studies (e.g., Dima et al. 2005), we followed in this work the conventional approach of decomposing the circulation into zonal-mean and eddy components. Given our findings that the eddy momentum transport is dominated by the gravest zonal component and can be largely understood as a zonal modulation of the symmetric Hadley cell, an alternative model of a nonzonal Hadley circulation (Schneider 1987; Hsu and Plumb 2000) may be preferable.

Acknowledgments. We are grateful to Sukyoung Lee and two anonymous reviewers for suggestions that improved this manuscript. P.Z.-G. acknowledges financial support by Grant CGL2015-72259-EXP by the State Research Agency of Spain. This work benefited from helpful discussions with I. Held during a series of summer visits to Princeton funded by NSF Grant AGS-1733818.

\section{REFERENCES}

Adames, Á. F., and J. M. Wallace, 2014: Three-dimensional structure and evolution of the MJO and its relation to the mean flow. J. Atmos. Sci., 71, 2007-2026, https://doi.org/ 10.1175/JAS-D-13-0254.1.
- , and —, 2017: On the tropical atmospheric signature of El Niño. J. Atmos. Sci., 74, 1923-1939, https://doi.org/10.1175/ JAS-D-16-0309.1.

,-- , and J. M. Monteiro, 2016: Seasonality of the structure and propagation characteristics of the MJO. J. Atmos. Sci., 73, 3511-3526, https://doi.org/10.1175/JAS-D-15-0232.1.

Andrews, D. G., and M. E. McIntyre, 1976: Planetary waves in horizontal and vertical shear: The generalized Eliassen-Palm relation and the mean zonal acceleration. J. Atmos. Sci., 33, 2031-2048, https://doi.org/10.1175/1520-0469(1976)033<2031: PWIHAV $>2.0 . \mathrm{CO} ; 2$.

- J. R. Holton, and C. B. Leovy, 1987: Middle Atmosphere Dynamics. International Geophysics Series, Vol. 40, Academic Press, 489 pp.

Bordoni, S., and T. Schneider, 2008: Monsoons as eddy-mediated regime transitions of the tropical overturning circulation. Nat. Geosci., 1, 515-519, https://doi.org/10.1038/ngeo248.

Caballero, R., and M. Huber, 2010: Spontaneous transition to superrotation in warm climates simulated by CAM3. Geophys. Res. Lett., 37, L11701, https://doi.org/10.1029/2010GL043468. , and H. Carlson, 2018: Surface superrotation. J. Atmos. Sci., 75, 3671-3689, https://doi.org/10.1175/JAS-D-18-0076.1.

Dee, D. P., and Coauthors, 2011: The ERA-Interim reanalysis: Configuration and performance of the data assimilation system. Quart. J. Roy. Meteor. Soc., 137, 553-597, https://doi.org/ 10.1002/qj.828.

Dima, I. M., J. M. Wallace, and I. Kraucunas, 2005: Tropical zonal momentum balance in the NCEP reanalyses. J. Atmos. Sci., 62, 2499-2513, https://doi.org/10.1175/JAS3486.1.

Gill, A., 1980: Some simple solutions for heat-induced tropical circulation. Quart. J. Roy. Meteor. Soc., 106, 447-462, https:// doi.org/10.1002/qj.49710644905.

Grise, K. M., and D. W. Thompson, 2012: Equatorial planetary waves and their signature in atmospheric variability. J. Atmos. Sci., 69, 857-874, https://doi.org/10.1175/JAS-D-11-0123.1.

Harnik, N., E. Heifetz, O. Umurhan, and F. Lott, 2008: A buoyancyvorticity wave interaction approach to stratified shear flow. J. Atmos. Sci., 65, 2615-2630, https://doi.org/10.1175/ 2007JAS2610.1.

Held, I. M., 1975: Momentum transport by quasi-geostrophic eddies. J. Atmos. Sci., 32, 1494-1497, https://doi.org/10.1175/ 1520-0469(1975)032<1494:MTBQGE > 2.0.CO;2.

Hide, R., 1969: Dynamics of the atmospheres of the major planets with an appendix on the viscous boundary layer at the rigid bounding surface of an electrically-conducting rotating fluid 
in the presence of a magnetic field. J. Atmos. Sci., 26, 841-853, https://doi.org/10.1175/1520-0469(1969)026<0841:DOTAOT> 2.0.CO;2.

Hoskins, B. J., and T. Ambrizzi, 1993: Rossby wave propagation on a realistic longitudinally varying flow. J. Atmos. Sci., 50, 1661-1671, https://doi.org/10.1175/1520-0469(1993)050<1661: RWPOAR $>2.0 . \mathrm{CO} ; 2$.

Hsu, C. J., and R. A. Plumb, 2000: Nonaxisymmetric thermally driven circulations and upper-tropospheric monsoon dynamics. J. Atmos. Sci., 57, 1255-1276, https://doi.org/10.1175/ 1520-0469(2000)057<1255:NTDCAU > 2.0.CO;2.

Iga, S. I., and Y. Matsuda, 2005: Shear instability in a shallow water model with implications for the Venus atmosphere. J. Atmos. Sci., 62, 2514-2527, https://doi.org/10.1175/JAS3484.1.

Ji, X., J. D. Neelin, S.-K. Lee, and C. R. Mechoso, 2014: Interhemispheric teleconnections from tropical heat sources in intermediate and simple models. J. Climate, 27, 684-697, https:// doi.org/10.1175/JCLI-D-13-00017.1.

Kraucunas, I., and D. L. Hartmann, 2005: Equatorial superrotation and the factors controlling the zonal-mean zonal winds in the tropical upper troposphere. J. Atmos. Sci., 62, 371-389, https:// doi.org/10.1175/JAS-3365.1.

- , and — 2007: Tropical stationary waves in a nonlinear shallow-water model with realistic basic states. J. Atmos. Sci., 64, 2540-2557, https://doi.org/10.1175/JAS3920.1.

Laraia, A. L., and T. Schneider, 2015: Superrotation in terrestrial atmospheres. J. Atmos. Sci., 72, 4281-4296, https://doi.org/10.1175/ JAS-D-15-0030.1.

Lee, S., 1999: Why are the climatological zonal winds easterly in the equatorial upper troposphere? J. Atmos. Sci., 56, 1353-1363, https://doi.org/10.1175/1520-0469(1999)056<1353:WATCZW> 2.0.CO;2.

Li, Y., J. Li, F. F. Jin, and S. Zhao, 2015: Interhemispheric propagation of stationary Rossby waves in a horizontally nonuniform background flow. J. Atmos. Sci., 72, 3233-3256, https:// doi.org/10.1175/JAS-D-14-0239.1.

Lindzen, R. S., and A. V. Hou, 1988: Hadley circulations for zonally averaged heating centered off the equator. J. Atmos. Sci., 45, 2416-2427, https://doi.org/10.1175/1520-0469(1988)045<2416: $\mathrm{HCFZAH}>2.0 . \mathrm{CO} ; 2$.

Lutsko, N. J., 2018: The response of an idealized atmosphere to localized tropical heating: Superrotation and the breakdown of linear theory. J. Atmos. Sci., 75, 3-20, https://doi.org/10.1175/ JAS-D-17-0192.1.

Pinto, J. R. D., and J. L. Mitchell, 2014: Atmospheric superrotation in an idealized GCM: Parameter dependence of the eddy response. Icarus, 238, 93-109, https://doi.org/10.1016/ j.icarus.2014.04.036.

Saravanan, R., 1993: Equatorial superrotation and maintenance of the general circulation in two-level models. J. Atmos. Sci., 50, 1211-1227, https://doi.org/10.1175/1520-0469(1993)050<1211: ESAMOT $>2.0 . \mathrm{CO} ; 2$.

Sardeshmukh, P. D., and B. J. Hoskins, 1988: The generation of global rotational flow by steady idealized tropical divergence. J. Atmos. Sci., 45, 1228-1251, https://doi.org/10.1175/ 1520-0469(1988)045<1228:TGOGRF>2.0.CO;2.

Schneider, E. K., 1987: A simplified model of the modified Hadley circulation. J. Atmos. Sci., 44, 3311-3328, https:// doi.org/10.1175/1520-0469(1987)044<3311:ASMOTM> 2.0.CO;2.

__ , and I. G. Watterson, 1984: Stationary Rossby wave propagation through easterly layers. J. Atmos. Sci., 41, 2069-2083, https://doi.org/10.1175/1520-0469(1984)041<2069:SRWPTE> 2.0.CO;2.

Shaw, T. A., 2014: On the role of planetary-scale waves in the abrupt seasonal transition of the Northern Hemisphere general circulation. J. Atmos. Sci., 71, 1724-1746, https://doi.org/ 10.1175/JAS-D-13-0137.1.

Showman, A. P., and L. M. Polvani, 2011: Equatorial superrotation on tidally locked exoplanets. Astrophys. J., 738, 71, https://doi.org/10.1088/0004-637X/738/1/71.

Suarez, M. J., and D. G. Duffy, 1992: Terrestrial superrotation: A bifurcation of the general circulation. J. Atmos. Sci., 49 , 1541-1554, https://doi.org/10.1175/1520-0469(1992)049<1541: TSABOT $>2.0 . \mathrm{CO} ; 2$.

Suhas, D., J. Sukhatme, and J. M. Monteiro, 2017: Tropical vorticity forcing and superrotation in the spherical shallow-water equations. Quart. J. Roy. Meteor. Soc., 143, 957-965, https:// doi.org/10.1002/qj.2979.

Vallis, G. K., 2006: Atmospheric and Oceanic Fluid Dynamics. Cambridge University Press, 745 pp.

Wang, P., and J. L. Mitchell, 2014: Planetary ageostrophic instability leads to superrotation. Geophys. Res. Lett., 41, 41184126, https://doi.org/10.1002/2014GL060345.

Zurita-Gotor, P., and P. Álvarez-Zapatero, 2018: Coupled interannual variability of the Hadley and Ferrel cells. J. Climate, $\mathbf{3 1}$, 4757-4773, https://doi.org/10.1175/JCLI-D-17-0752.1.

__ , and I. M. Held, 2018: The finite amplitude evolution of mixed Kelvin-Rossby wave instability and equatorial superrotation in a shallow-water model and an idealized GCM. J. Atmos. Sci., 75, 2299-2316, https://doi.org/10.1175/ JAS-D-17-0386.1. 